\title{
How to increase precision in Xenopus gene editing and base editing experiments.
}

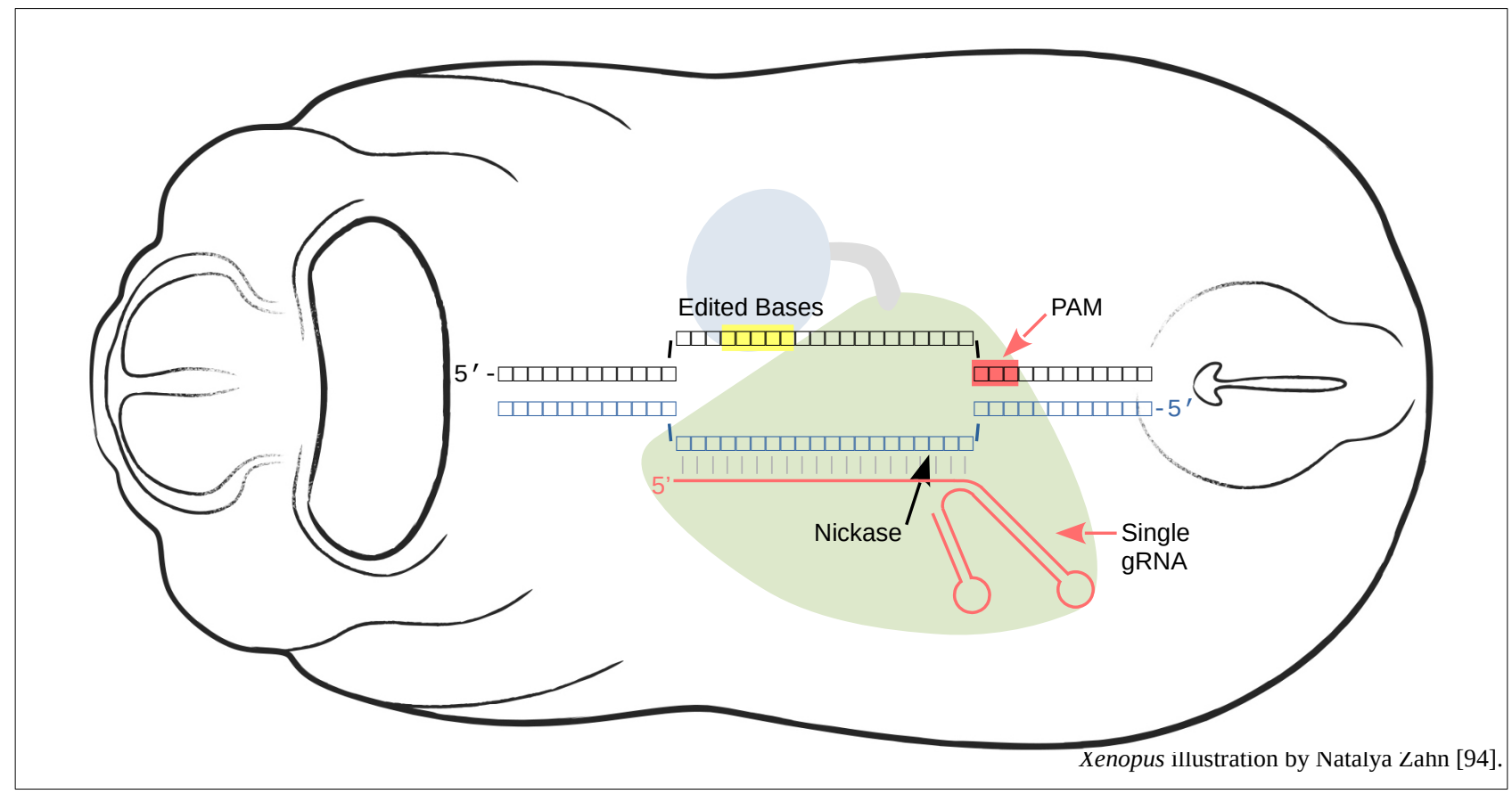

Stuart J. Smith.

ORCiD: 0000-0001-7266-6790.

Email: <stuart.smith@adprhl1.org>

\section{Summary.}

A review focussed on improvements in genome editing methods for developmental biology research using aquatic vertebrate embryos from Xenopus frog species. It is aimed as an introduction for students learning the differences between CRISPR/Cas9-mediated gene mutation versus base editing and prime editing strategies. The benefits, limitations and efficiencies achieved by the different methods are discussed.

\section{Contents.}

1. Introduction.

2. Pros and cons of the Xenopus embryo.

3. Published Xenopus CRISPR/Cas9 research - Can the mosaicism that results from repair of Cas9 DSBs be eliminated?

4. CRISPR/base editing for precise transition mutations in genes.

5. CRISPR/base editing in Xenopus embryos.

6. CRISPR/prime editing for transversion mutations, accurate small insertions and deletions.

7. Methods to save a generation during Xenopus gene mutation studies.

8. DSB repair options for larger DNA sequence insertions in Xenopus embryos.

9. $X$. laevis and $X$. tropicalis are suited to different types of gene editing projects (at the moment).

10. The education of gene editing - A new role for $X$. laevis embryos.

11. Concluding remarks.

\section{Keywords.}

Xenopus; CRISPR/Cas9; CRISPant; base editor; ABE; CBE; prime editor; review. Short title: Xenopus CRISPR experimental strategies. 


\section{Introduction.}

CRISPR (clustered regularly interspaced short palindromic repeats)/Cas9 technology is redefining what is possible in biomedical research through the use of reverse genetics and genome editing. The key to its success is the simplicity by which the Cas9 endonuclease can be programmed to cut a double-strand DNA break (DSB) at a specific sequence when complexed with a short, single-guideRNA (gRNA). Some compelling research applications of CRISPR/Cas9 are seen in experiments with aquatic vertebrate embryos, including those from Xenopus frog species. In its simplest guise, injection of Cas9 and a gRNA into newly fertilized embryos can specifically target the mutation of a gene and facilitate the immediate discovery of defective developmental phenotypes that arise as a result. These G0-generation mutated embryos are cheap to produce and valuable for confirming previous antisense morpholino gene knockdown results, but there are often limits to the level of phenotypic detail they can provide. They are often referred to as CRISPant embryos (just as morpholino embryos are known as morphants), or F0-generation mutations (F, filial).

While Cas9 will cut at the same site in each copy of the targeted gene, the error-prone mechanism through which the genome is subsequently repaired by non-homologous end-joining (NHEJ) results in a variety of different sequence lesions, including base insertions, deletions (indels) and missense mutations. DSB formation and emergency repair occurs simultaneously with ongoing DNA synthesis and cell division, so mutated alleles can have a mosaic distribution among cells as an animal grows. It is this variation in gene mutations occurring across cohorts of injected embryos and the cellular mosaicism within individuals that makes quantitative analysis of abnormal developmental morphologies difficult. The different mutations will not all effect the function of the targeted gene to the same degree and so a spectrum of phenotype severities is observed. The standard reverse genetic approach to resolve such variability is to commit to a lengthy breeding programme; reduce the initial efficiency of gene mutation, raise embryo carriers to adulthood, select defined mutations for study from F1-progeny and obtain the phenotype of homozygous recessive deleterious alleles at the F2-generation or later. But with CRISPR experiments in Xenopus embryos, alternative strategies are now possible that aim to achieve the same outcome; methods that increase the precision of gene editing and thus maintain the traditional virtues of rapid studies, large scale and low cost.

In this review, I have emphasized recent work that has progressed beyond the basic production of targeted gene mutations in Xenopus using CRISPR/Cas9-induced DSBs. Themes discussed include: how to minimize cellular mosaicism and phenotypic variation; the emergence of base editors, prime editors and their suitability for Xenopus studies; embryological methods to speed up phenotype discovery; plus strategies for inserting longer sequences into genes. I have included data on the relative efficiencies achieved by the different methods, where available, so that researchers can make an informed choice on the likelihood of success when designing their own experiments. The final section publicises efforts to incorporate CRISPR/Cas9-mediated gene mutation in Xenopus embryos into education programmes to provide the next generation of scientists and clinicians with invaluable practical experience of genome editing.

\section{Pros and cons of the Xenopus embryo.}

Amphibian embryos like Xenopus have consistently provided major insights across all scales of biological research (see www.xenbase.org/anatomy/intro.do, RRID:SCR_003280) [1,2]. Their prolific spawn and external development, the large embryo size of $X$. laevis (1.4 mm) and broad repertoire (plus famed tolerance) of experimental manipulations are well-proven (Table 1). Xenopus has a closer evolutionary relationship with mammals compared to other aquatic model species like zebrafish. Amphibian evolution diverged from the amniote lineage some 360 million years ago [Reviews 3-5] and they share many terrestrial adaptations beyond the four limbs and lungs that 
form during feeding tadpole stages. The embryonic heart, for example, has acquired a partial separation of the systemic and pulmonary blood flows [6]. Xenopus forms a three-chambered heart with fully septated atria and featuring a single large ventricle containing patterns of myofibril assembly [7] that are relevant to the high pressure hearts of mammals and birds.

\section{Table 1. Historical strengths and uses of Xenopus embryos.}

Controlled over-expression or knockdown of gene activity within defined tissues, taking advantage of the early embryo cell-fate map to target injection of mRNA, protein or antisense reagents

Stable integration of transgenes for lineage-restricted synthesis of fluorescent markers and recombinant proteins

Perturbation of regulatory networks with dominant-negative, or positively acting protein variants

Surgical grafting of tissue transplants and powerful explant culture assays

An abundant tissue and protein source for high-throughput biochemical study

All these experiments are simple to achieve in Xenopus and are frequently combined into detailed explorations of gene and protein function

High quality genome assemblies are available for the widely used $X$. laevis species [8], an allotetraploid, and the smaller diploid relative $X$. tropicalis [2,9]. Their chromosomes display long stretches of gene colinearity with mammals and birds, this synteny underpinning the often quoted report that $83 \%$ of identified human disease genes have a verified ortholog in Xenopus [2,9]. One perceived weakness of Xenopus as an experimental model is the time needed for the frogs to reach sexual maturity (12 months/6 months for $X$. laevis/tropicalis). Breeding programmes that reveal genotype/phenotype relationships for homozygous gene mutations are possible only with $X$. tropicalis [Review 10], but still represent a significant time and financial commitment.

Nevertheless, technology based upon CRISPR/Cas9 [11] is proving to be transformative for Xenopus research. The targeted disruption of all copies of a gene is now possible in one-cell stage embryos, with instant readout of the developmental consequences. CRISPR/base editors may prove even more powerful. They accurately introduce specific point mutations without mosaicism, without the genomic damage associated with DSBs and with an efficiency that obviates the need for multi-generation transmission of the mutated allele. Prime editors will install mutations, or defined insertions and deletions. The new methods promise to change the scope of Xenopus embryo studies.

\section{Published Xenopus CRISPR/Cas9 research - Can the mosaicism that results from repair of Cas9 DSBs be eliminated?}

Many published CRISPR/Cas9 studies have successfully obtained consistent developmental phenotypes in Xenopus after targeted gene mutation in founder, G0-generation animals (for both $X$. laevis and tropicalis, see Table 2). Not all of these have fully documented the underlying DNA sequence variation at the DSB site. Some did use next generation sequencing (NGS) [12-14] or Sanger sequencing of cloned genomic isolates from the embryos [14-19]. Others merely monitored the breakdown of Sanger traces from directly amplified DNA at the target site, combined with a deconvolution algorithm to predict likely repairs [20,21]. Indels that cause reading frame-shifts are a frequent outcome, so analyses with basic DNA genotyping have instead emphasized the depletion of the targeted protein measured with specific antibody staining [21-23]. Reported mutation efficiencies have varied, which probably reflects differing Cas9-gRNA reagent activity. The widespread practice of substituting-in a mismatched G-nucleobase at the 5'-end of the gRNA to facilitate its synthesis by in vitro transcription from a DNA template may have caused some dropoff in efficacy (Table 2). As an alternative, synthetic production of gRNA is now commercially available. While it is promoted as a means to increase editing efficiency in cultured cells, synthetic production can also provide greater freedom during gRNA design while maintaining $100 \%$ sequence complementarity. 


\begin{tabular}{|c|c|c|c|c|c|c|c|c|c|c|c|c|c|c|c|c|c|c|c|c|c|c|}
\hline 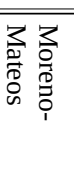 & 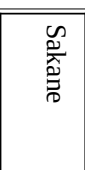 & 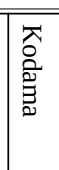 & $\frac{1}{\tilde{0}}$ & 胥 & & & ż. & 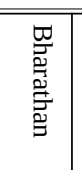 & 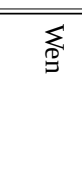 & $\begin{array}{l}\forall \\
\frac{\mathscr{\sigma}}{\sigma}\end{array}$ & 总 & 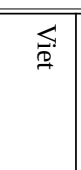 & 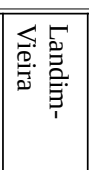 & 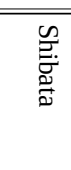 & & & 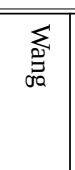 & $E$ & & & $\overline{\tilde{\sigma}}$ & 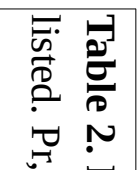 \\
\hline 产 & 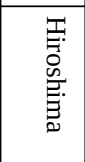 & \begin{tabular}{|l|}
$\overrightarrow{0}$ \\
층
\end{tabular} & 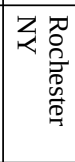 & 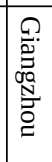 & & & 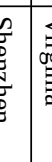 & 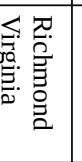 & 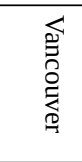 & $\begin{array}{l}\overrightarrow{\widetilde{\sigma}} \\
\tilde{\sigma}\end{array}$ & $\begin{array}{l}\stackrel{Q}{\vec{\sigma}} \\
\stackrel{\overrightarrow{0}}{g}\end{array}$ & 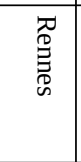 & $\frac{\vec{\sigma}}{\sigma}$ & 学 & & & 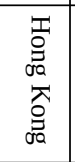 & 总 & & & & 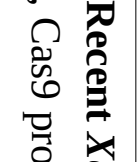 \\
\hline$\stackrel{\widetilde{G}}{v}$ & $\begin{array}{l}\tilde{0} \\
\stackrel{\infty}{0}\end{array}$ & $\underset{\infty}{\stackrel{\sim}{0}}$ & $\underset{\infty}{\tilde{O}}$ & $\underset{\infty}{\tilde{O}}$ & & & $\vec{b}$ & 论 & $\underset{0}{\tilde{0}}$ & $\begin{array}{l}\tilde{O} \\
\theta\end{array}$ & $\stackrel{\tilde{0}}{\tilde{0}}$ & $\begin{array}{l}\tilde{D} \\
\tilde{O}\end{array}$ & $\begin{array}{l}\tilde{D} \\
\tilde{O}\end{array}$ & 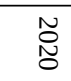 & & & $\begin{array}{l}\tilde{D} \\
\text { O }\end{array}$ & $\begin{array}{l}\tilde{D} \\
\text { N }\end{array}$ & & & & \\
\hline 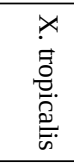 & 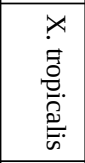 & 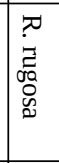 & 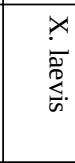 & 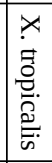 & & & 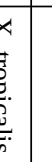 & 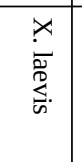 & 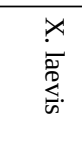 & 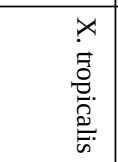 & 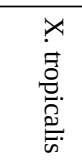 & 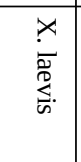 & 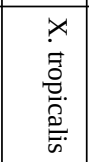 & 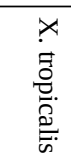 & & & 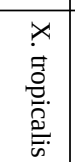 & 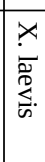 & $x$ & & 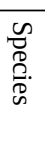 & 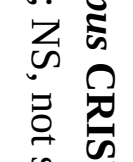 \\
\hline 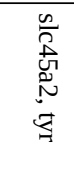 & 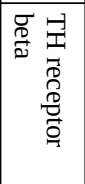 & 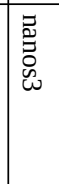 & 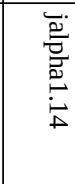 & 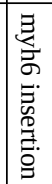 & 践 & & ș & क्षि & 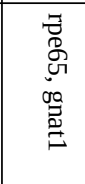 & 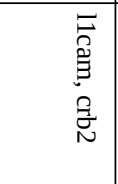 & $\vec{x}$ & 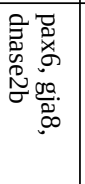 & 志 & 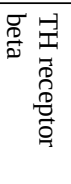 & & & 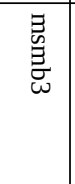 & 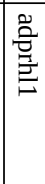 & $\exists$ & & 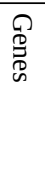 & 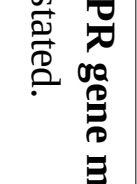 \\
\hline 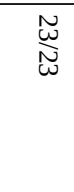 & 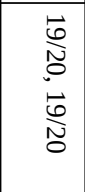 & \begin{tabular}{|l|}
\multirow{O}{O}{} \\
Oे
\end{tabular} & $\begin{array}{l}\overrightarrow{0} \\
\text { Nै }\end{array}$ & $\begin{array}{l}\text { No } \\
\text { No }\end{array}$ & & & : & $\begin{array}{l}\tilde{N} \\
\mathbb{N} \\
\mathbb{0} \\
\tilde{N} \\
\mathbb{N} \\
0\end{array}$ & $\begin{array}{l}\text { N } \\
\stackrel{N}{0} \\
\stackrel{0}{N} \\
\stackrel{N}{0}\end{array}$ & 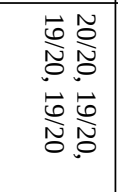 & 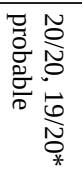 & 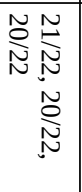 & 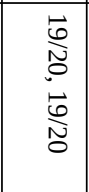 & $\begin{array}{l}\overrightarrow{0} \\
\stackrel{0}{0}\end{array}$ & & & 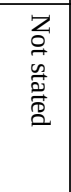 & 芯 & & & 初 & 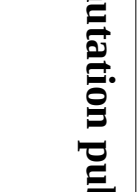 \\
\hline 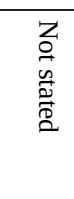 & $\begin{array}{l}\vec{p} \\
\dot{\phi} \\
\dot{\phi}\end{array}$ & 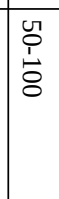 & 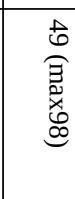 & $\begin{array}{l}\stackrel{9}{\mathrm{~V}} \\
\mathrm{i}\end{array}$ & $\bar{\sigma}$ & 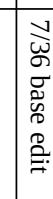 & & 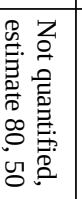 & 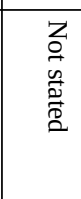 & 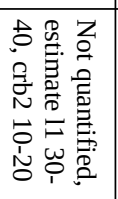 & 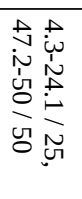 & $\begin{array}{l}\vec{b} \\
0 \\
o \\
o \\
\text { c }\end{array}$ & 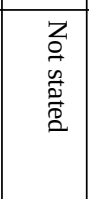 & 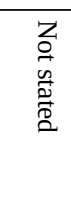 & & & $\tilde{v}$ & 호 & & & 愙 & \\
\hline 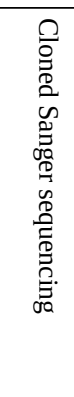 & $\begin{array}{l}\text { Za } \\
\text { w }\end{array}$ & 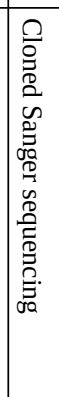 & 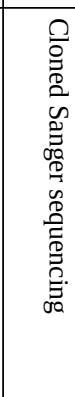 & 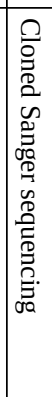 & $\begin{array}{l}0 \\
0 \\
0 \\
0 \\
0 \\
0 \\
0 \\
0 \\
0 \\
0 \\
0 \\
0 \\
0 \\
0 \\
0 \\
0 \\
0\end{array}$ & 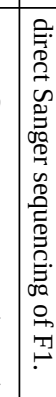 & & 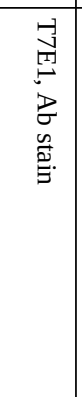 & 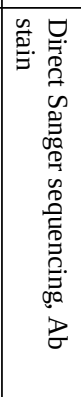 & 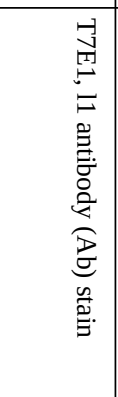 & 公 & 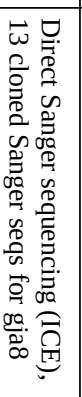 & 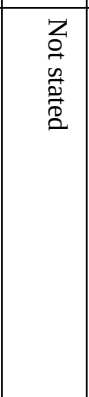 & $\begin{array}{l}z \\
a \\
0 \\
0 \\
0 \\
0 \\
0 \\
0\end{array}$ & & & 검 & 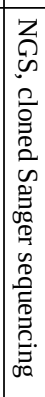 & & & 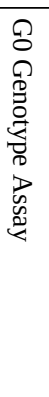 & \\
\hline 8 & 8 & 8 & 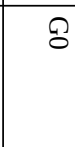 & Q & a & & 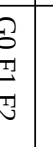 & 8 & 8 & $\Omega$ & 8 & 8 & 8 & $\begin{array}{l}8 \\
8 \\
\text { T囗⿱ } \\
\text { 정 }\end{array}$ & & & 8 & 8 & & & $\begin{array}{l} \\
0 \\
0 \\
0 \\
0 \\
0 \\
0 \\
0 \\
0\end{array}$ & \\
\hline 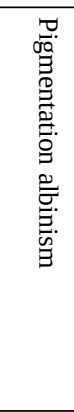 & 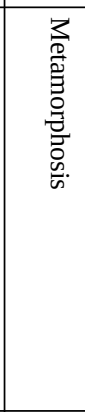 & 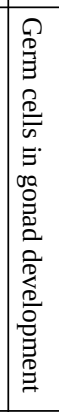 & 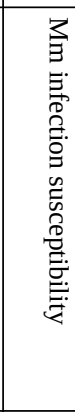 & 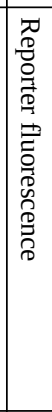 & 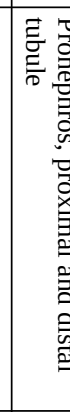 & & 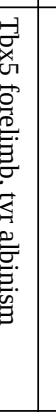 & 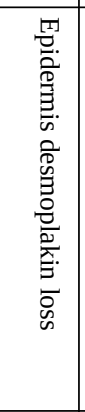 & 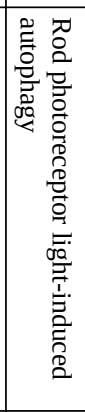 & 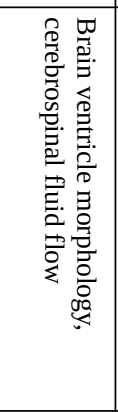 & 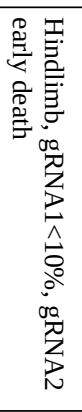 & 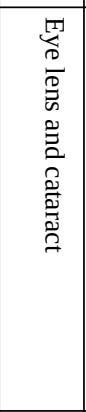 & 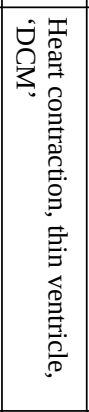 & 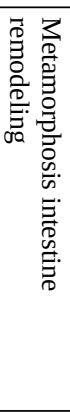 & 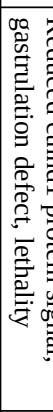 & & 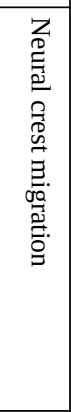 & 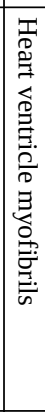 & & & 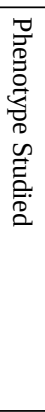 & 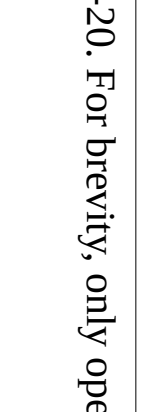 \\
\hline $\begin{array}{l}0 \\
\stackrel{0}{0} \\
\dot{1} \\
0 \\
=\end{array}$ & 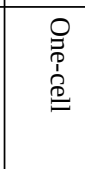 & 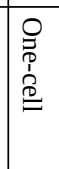 & $\begin{array}{l}\stackrel{0}{9} \\
\stackrel{p}{1} \\
0 \\
=\end{array}$ & 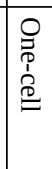 & 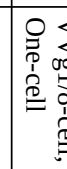 & & 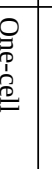 & $\begin{array}{l} \\
\stackrel{0}{p} \\
\dot{1} \\
\underline{D} \\
\end{array}$ & $\begin{array}{l}\stackrel{\rho}{P} \\
\stackrel{p}{1} \\
\stackrel{0}{=}\end{array}$ & $\begin{array}{l}0 \\
\stackrel{0}{0} \\
\dot{1} \\
\stackrel{0}{=}\end{array}$ & 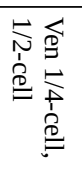 & $\begin{array}{l}0 \\
0 \\
0 \\
1 \\
0 \\
=\end{array}$ & $\begin{array}{l} \\
\stackrel{0}{0} \\
\dot{1} \\
\underline{0} \\
\end{array}$ & $\begin{array}{l}\stackrel{\bigcirc}{9} \\
\stackrel{p}{1} \\
\stackrel{0}{=}\end{array}$ & 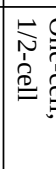 & & 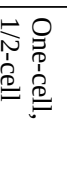 & $\begin{array}{l}0 \\
0 \\
0 \\
1 \\
0 \\
0 \\
\end{array}$ & & & & \\
\hline 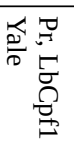 & 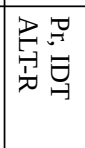 & 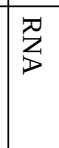 & 帝 & $\prod_{\Delta}^{\pi}$ & 然 & 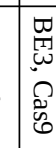 & & 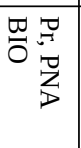 & Z & 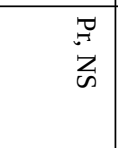 & 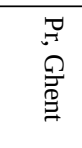 & ت্ت & $\begin{array}{l}\overrightarrow{0} \\
z \\
m\end{array}$ & Z & & & 焉营 & 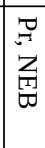 & & & & \\
\hline 苞 & 坖 & $\ddot{\mathscr{\Xi}}$ & $\vec{\sigma}$ & 3 & - & & $\infty$ & $\underset{\sim}{\mathbb{N}}$ & $\stackrel{\mathbb{N}}{ }$ & 胥 & $\underline{\omega}$ & $\vec{\sigma}$ & $\begin{array}{l}\underset{\infty}{\infty} \\
\stackrel{\infty}{\infty}\end{array}$ & $\mathcal{J}$ & & & $\overline{\mathscr{\sigma}}$ & \pm & & & s & \\
\hline
\end{tabular}


The introduction mentioned that the variation and mosaicism of gene mutations found in CRISPant embryos can present a challenge to the quantitative analysis of phenotype traits. My own work provides a useful example. When I reported defective heart chamber outgrowth caused by knockout of ADP-ribosylhydrolase-like 1 (adprhl1) gene function, the range of ventricle abnormalities observed in tadpoles needed careful classification [14]. Moreover, within each heart, the severity of myofibril assembly defects differed appreciably, even amongst adjacent cardiomyocytes within the ventricle wall. Cardiomyocytes that lacked any myofibrils were attributed to the adprhl1-null functional state; moderately affected cells with mis-oriented myofilaments likely contained a mutated gene copy with partial activity. But there is no easy way to confirm this or to apportion cell morphologies to particular hypomorphic mutations within a complex genetically mosaic animal. Plus, alternative interpretations cannot be ruled out. Thus as the resolution of analysis is increased, the inherent variability of CRISPant embryos becomes unsatisfactory, and similar limitations are also seen in other recent publications.

Uniformity of phenotype can be achieved in two ways, by taking steps to ensure all the mosaic mutations are complete functional-nulls at the protein level, or preferably by eliminating the mosaicism entirely. A stable, measurable mutant defect will always provide clearer information on the function of a gene within a complex developmental process. Likewise, replicating the aetiology of a human genetic disease in an animal model depends on installation of specific gene mutations. In short, gene editing in Xenopus needs greater precision.

To increase the percentage of functional-null mutations, more nuanced positioning of the gRNA within the gene's coding sequence can be of benefit. Early studies tended to select gRNAs near the 5 '-start of the coding sequence to aim for non-functional, frame-shift mutations (a valid approach when specific mutations are then selected in subsequent generations). However during DSB repair, some indels that preserve the correct reading frame will inevitably occur in every injected embryo. Such 5'-located in-frame mutations may well retain protein activity and if so, consistent phenotypes are difficult to achieve. Targeting 5'-frame-shifts can also be complicated by distinct promoter use, (in-frame) exon skipping [24,25], or the mutations unmasking alternative downstream translation initiation sites. There is now greater appreciation during gRNA design that a hybridization site that encodes a key part of a protein structure [26], or an essential functional domain [27,see PAVOOC gRNA design tool 28], might be a better prospect for G0-phenotype discovery. This increases the chance that the in-frame mutations will also be defective due to protein instability or loss of activity. It should be noted that with this targeting strategy, the researcher does need to be alert to the possibility of dominant effects arising from the in-frame mutations.

The second option, the rapid production of embryo cohorts carrying uniformly mutated alleles, must be the goal for founder generation studies. NHEJ of DSBs does not produce a completely random selection of mutations, as the local DNA sequence either side of the cut influences the type of repairs that occur. Short runs of repeated sequence impose a marked bias to favour end-joining at the microhomology sites [29,30], with deletion of the intervening bases. Computational methods trained on actual repairs in mouse ES cells have now been used to predict accurately the frequency of occurrence of mutated variants observed in frog and fish embryos [31]. This could facilitate another type of gRNA design that selects a target site where a single given frame-shift mutation will be the predominant outcome (or at least, where in-frame repairs will not occur). It remains to be seen whether refinements to gRNA design can truly eliminate the mosaicism associated with DSB repair. Instead, these strategies are likely to be eclipsed by base editing and prime editing technology, which does not rely on DSB cleavage and offers far greater control for production of defined point mutations. 


\section{CRISPR/base editing for precise transition mutations in genes.}

Base editors bring together the activities of several enzymes to execute the conversion of one DNA nucleobase into another at a defined position within a targeted sequence. The two classes described thus far perform cytosine to thymine (a $\mathrm{C}: \mathrm{G}$ to a T:A pair) or adenine to guanine (A:T to G:C pair) transition mutations (pyrimidine to pyrimidine, or purine to purine conversions).

Cytosine base editors (CBE) initially convert the base cytosine to uracil. Komor et al [32] built their CBE in three stages, exploiting the cytidine deaminase enzyme rAPOBEC1 because it will act on single-stranded DNA as well as RNA as a substrate [33]. The first-generation CBE (BE1) fused rAPOBEC1 to the catalytically inactive dCas9 using a 16 aa XTEN-based linker and proved active in vitro. The length of linker confers the distance, or activity window, where the cytidine deaminase reaction can occur. Upon hybridization of a BE1-gRNA complex to its target sequence, the displaced DNA strand (the protospacer adjacent motif (PAM) containing strand) becomes accessible to the enzyme. Any cytosine present at positions 4 to 8 on the displaced strand (counted from the 5'end of the gRNA sequence, so distal to the PAM) can be modified to uracil.

For use in cells and in vivo, a further addition to the CBE was produced to prevent the cellular DNA repair response from correcting the mismatched $\mathrm{U}: \mathrm{G}$ base pair product, plus a nuclear localization signal (NLS) was added to the C-terminus. Uracil DNA glycosylase normally catalyses the removal of $U$ from DNA in cells and initiates base-excision repair [Review 34], which would revert U:G back to a C:G pair. Hence, the second-generation CBE (BE2) included a small uracil DNA glycosylase inhibitor (UGI) protein from $B$. subtilis bacteriophage PBS1 [35] to block any baseexcision at the target site [32]. A third refinement to the CBE (BE3) seeks to replace the corresponding base on the non-edited DNA strand so as to pair with the edited base of the displaced strand (from $U: G$ to $U: A$ ), thus increasing the proportion of edited sequences propagated (as T:A) through subsequent rounds of DNA replication. BE3 mimics the process of mismatch repair, where nicks are used to mark the presence of errors in newly synthesized DNA strands, which are then removed and resynthesized. BE3 has switched to the D10A nickase version of Cas9 (rAPOBEC1XTEN-Cas9(D10A)-UGI-NLS) [32]. This nickase has the HNH nuclease domain intact (H840), the RuvC nuclease domain inactive (D10A), so makes a nick in the strand of target DNA that hybridizes with the gRNA (the opposite strand to the base edited strand). Tested extensively in human cell cultures, BE2 achieved a 20\% C to T conversion efficiency of DNA sequences at gRNA-defined positions with minimal $\leq 0.1 \%$ occurrence of indels. BE3 conversion efficiency increased to $37 \%$, at the expense of $1.1 \%$ indel frequency on average [32]. At the same time, an alternative CBE named Target-AID was developed independently using the PmCDA1 cytidine deaminase [36].

Adenine base editors (ABE) have been produced [37]. They utilize an adenosine deaminase (ecTadA) that would ordinarily act on transfer-RNA during formation of a hypoxanthine base (=inosine nucleoside, I) for wobble pairing [38]. Natural TadA does not accept DNA as a substrate. Consequently, the ABE construction strategy employed directed evolution in bacteria and protein engineering to mutate TadA so that it acted on single-stranded DNA when part of a dCas9 fusion. The assembled ABE (ABE7.10) combined a dimer of wild-type and evolved TadA with the Cas9 nickase (wtTadA-32aa-TadA*-32aa-Cas9(D10A)-NLS) [37]. Inosine is read as G by polymerases, so a targeted A:T base pair that is converted to the mismatched I:T, then I:C, will be propagated as G:C after DNA replication. ABE7.10 has shown remarkable activity. Tested with many gRNAs in cell culture, A to $\mathrm{G}$ base conversion efficiency averaged 53\% (max 68\%), with indels and non-G base edits rarely occurring at $\leq 0.1 \%$ [37]. Once converted, there appears to be little endogenous activity (in human cells) to remove inosine from DNA, which underscores both the high activity and precision of base editing observed with ABE7.10. 
After the initial publications, and through 2020, many further improvements to both CBEs and ABEs have been reported. Tables 3 and 4 list new base editors to illustrate the rapid pace of development. The aim has been to increase activity and precision, offer a choice of different target specificities by using Cas nuclease homologs with alternate PAM requirements, while eliminating undesirable off-target mutagenic activity. Briefly: rapid evolution of the deaminase components has improved the CBE performance on GC substrates and ABE compatibility with Cas homologs; increased UGI activity for the CBE to suppress base-excision, which was a source of DSBs; a simplified ABE architecture that dispenses with the fused TadA dimer; linker lengths adjusted, the NLS and codon usage optimized for better expression yields. These state of the art editors (Tables 3 , 4 highlighted) routinely achieve $60-80 \%$ base conversion in cultured cells, the maximum simply reflecting limitations of DNA transfection protocols or residual cellular DNA repair. Comparable efficiency is observed at all positions across their activity window [39].

Base editors still have some downsides. Unwanted off-target activity can be divided into three types. Cas9-dependent off-target DNA editing activity is much like the original Cas9 nuclease. Guide-RNA sequences must be screened for potentially problematic hybridization to other regions of the genome and putative off-target sites then assessed for the presence of editable bases in their activity window. Supplying the base editor as a protein-gRNA complex rather than in DNAencoded form greatly reduces off-targeting at sites where complementarity with the gRNA is less than 100\% [40,41]. Cas9-independent off-target DNA editing by CBEs has been shown to occur at very low frequency [42-44]. It results from the binding affinity of the cytidine deaminase component for single-stranded DNA, prefers TCW targets but shows no broader sequence specificity, nor any bias towards particular genomic regions [44]. Assays have already identified which of the improved CBEs exhibit reduced Cas9-independent off-target activity [45]. ABEs rely more strictly on being guided to a DNA target before any base editing can occur. Finally, transcriptome-wide off-target base deamination within cellular RNA has been detected in transfected cells expressing CBEs or ABEs [46,47]. This would be a concern for scenarios where base editors are supplied and remain active for an extended period, so again, engineered forms with reduced RNA editing capability have been produced [CBE 46,47] [ABE 41,47,48].

Base editors are not yet ready for human therapeutic use, but have been quickly adopted for installing transition mutations in many laboratory animal and plant species [Review 49]. As cytosine base editing was developed first, it has seen the most use, including in zebrafish $[40,50]$, Xenopus [51,18] and mouse embryos [52,53]. Moreover, base editors have also been delivered into discrete tissues within mice to demonstrate their potential to correct mutations and treat genetic disease models [40,54,55]. For these adult treatment regimes, a choice of delivery vectors are available, with either localized or systemic administration routes. For example, supply of ABEs into mice has been in DNA-encoded form, as plasmid [systemic 56], or as dual adeno-associated viruses via an intein-split system [localized 54,57] [systemic 58,55], or as a mRNA containing chemical modifications to prolong its lifespan [systemic 59]. 
Table 3. List of cytosine base editors. Chronological production of CBEs by David R. Liu's lab.

\begin{tabular}{|c|c|c|c|c|c|}
\hline \multicolumn{4}{|l|}{ Cytosine base editors } & \multicolumn{2}{|l|}{ Abbreviation: evo', evolution } \\
\hline $\begin{array}{l}\text { Cytosine base editor name / } \\
\text { Architecture }\end{array}$ & NLS & $\begin{array}{l}\text { Codon } \\
\text { optimize }\end{array}$ & $\begin{array}{l}\text { Activity } \\
\text { window }\end{array}$ & Features & Ref \\
\hline $\begin{array}{l}\text { Target-AID (produced by the Kondo lab) } \\
\text { NLS-Cas9(D10A)-NLS-CDA1-NLS-UGI }\end{array}$ & SV40 & No & $2-4$ & An alternative $\mathrm{CBE}$ & {$[36]$} \\
\hline $\begin{array}{l}\text { BE3 } \\
\text { rAPOBEC1-XTEN16aa-Cas9(D10A)-4aa-UGI-NLS }\end{array}$ & SV40 & $\begin{array}{l}\text { No } \\
\text { (IDT) }\end{array}$ & $4-8$ & Original paper, 3rd generation & {$[32]$} \\
\hline $\begin{array}{l}\text { BE4 } \\
\text { rAPOBEC1-32aa-Cas9(D10A)-9aa-UGI-9aa-UGI-NLS }\end{array}$ & SV40 & No & $4-8$ & $\begin{array}{l}\text { Improved conversion efficiency } \\
\text { Improved base edit purity }\end{array}$ & {$[99]$} \\
\hline $\begin{array}{l}\text { BE4-Gam / Gam-16aa- rAPOBEC1-32aa-Cas9(D10A)- } \\
\text { 9aa-UGI-9aa-UGI-NLS }\end{array}$ & SV40 & No & $4-8$ & Reduced indel formation & {$[99]$} \\
\hline $\begin{array}{l}\text { BE4max / bpNLS-rAPOBEC1-32aa-Cas9(D10A)-9aa- } \\
\text { UGI-9aa-UGI-bpNLS }\end{array}$ & $\begin{array}{l}\text { 2xbi- } \\
\text { partite }\end{array}$ & $\begin{array}{l}\text { Yes-Ge- } \\
\text { nScript }\end{array}$ & $(3) 4-8$ & $\begin{array}{l}\text { Improved protein expression and } \\
\text { localization in mammalian cells }\end{array}$ & {$[100]$} \\
\hline $\begin{array}{l}\text { AncBE4max / bpNLS-APOBEC*-32aa-Cas9(D10A)- } \\
\text { 9aa-UGI-9aa-UGI-bpNLS }\end{array}$ & $\begin{array}{l}\text { 2xbi- } \\
\text { partite }\end{array}$ & $\begin{array}{l}\text { Yes-Ge- } \\
\text { nScript }\end{array}$ & $(3) 4-8$ & $\begin{array}{l}\text { Ancestral sequence } \\
\text { reconstruction (ASR, Anc689), } \\
\text { improved protein expression }\end{array}$ & {$[100]$} \\
\hline $\begin{array}{l}\text { evoAPOBEC1-BE4max / bpNLS-APOBEC1*- } \\
\text { 32aa-Cas9(D10A)-9aa-UGI-9aa-UGI-bpNLS }\end{array}$ & $\begin{array}{l}\text { 2xbi- } \\
\text { partite }\end{array}$ & $\begin{array}{l}\text { Yes-Ge- } \\
\text { nScript }\end{array}$ & $3-8$ & $\begin{array}{l}\text { Evolved APOBEC1 for reduced } \\
\text { target bias at edge of window } \\
\text { Now edits GC targets efficiently } \\
\text { Phage-assisted continuous evo' }\end{array}$ & {$[39]$} \\
\hline $\begin{array}{l}\text { evoFERNY-BE4max / bpNLS-APOBEC**-32aa- } \\
\text { Cas9(D10A)-9aa-UGI-9aa-UGI-bpNLS }\end{array}$ & $\begin{array}{l}\text { 2xbi- } \\
\text { partite }\end{array}$ & $\begin{array}{l}\text { Yes-Ge- } \\
\text { nScript }\end{array}$ & $3-8$ & $\begin{array}{l}\text { Evolved smaller ASR Anc656 } \\
\text { variant, edits GC targets }\end{array}$ & {$[39]$} \\
\hline $\begin{array}{l}\text { evoCDA1-BE4max / bpNLS-CDA1*-32aa- } \\
\text { Cas9(D10A)-9aa-UGI-9aa-UGI-bpNLS }\end{array}$ & $\begin{array}{l}\text { 2xbi- } \\
\text { partite }\end{array}$ & $\begin{array}{l}\text { Yes-Ge- } \\
\text { nScript }\end{array}$ & $\begin{array}{l}3-12 \\
(13)\end{array}$ & $\begin{array}{l}\text { Evolved CDA1 deaminase with } \\
\text { broad activity window }\end{array}$ & {$[39]$} \\
\hline \multicolumn{6}{|c|}{ Cytosine base editors with altered PAM specificity or activity window size } \\
\hline SaBE3, SaBE3-Gam, SaBE4, SaBE4-Gam & \multicolumn{2}{|c|}{ As BE3, BE4 } & Broader & PAM is NNGRRT & $\begin{array}{l}{[99,1} \\
01]\end{array}$ \\
\hline $\begin{array}{l}\text { SaKKH-BE3, VQR-BE3, EQR-BE3, VRER- } \\
\text { BE3 }\end{array}$ & \multicolumn{2}{|c|}{ As BE3 } & $\begin{array}{l}\text { SaKKH } \\
\text { broader }\end{array}$ & $\begin{array}{l}\text { PAMs are NNNRRT, NGA, } \\
\text { NGAG, NGCG respectively }\end{array}$ & [101] \\
\hline xCas9-BE3, xCas9-BE4 & \multicolumn{2}{|c|}{ As BE3, BE4 } & $4-8$ & $\begin{array}{l}\text { Allowed PAMs are NG/GAA/ } \\
\text { GAT(NGNC), evolved SpCas9 }\end{array}$ & {$[102]$} \\
\hline $\begin{array}{l}\text { evoAPOBEC1-BE4max-NG / bpNLS- } \\
\text { APOBEC1*-32aa-Cas9-NG(D10A)-9aa-UGI-9aa-UGI- } \\
\text { bpNLS }\end{array}$ & \multicolumn{2}{|c|}{$\begin{array}{l}\text { As } \\
\text { evoAPOBEC1- } \\
\text { BE4max }\end{array}$} & $3-8$ & $\begin{array}{l}\text { PAM is NGH (lower NGC, tested } \\
\text { with AGT), evolved APOBEC1 } \\
\text { with engineered SpCas9 } \\
\text { All evo-editors available as -NG }\end{array}$ & $\begin{array}{l}{[39,1} \\
03]\end{array}$ \\
\hline YE1-BE3, YE2-BE3, EE-BE3, YEE-BE3 & \multicolumn{2}{|c|}{ As BE3 } & $5-6$ & $\begin{array}{l}\text { Narrow activity window, } \\
\text { APOBEC1 catalytic mutations }\end{array}$ & {$[101]$} \\
\hline $\begin{array}{l}\text { CP1012-CBEmax, also CP1028-, CP1041-, } \\
\text { CP1249- }\end{array}$ & \multicolumn{2}{|c|}{ As BE4max } & $\begin{array}{l}4-11 \\
\text { to }-13\end{array}$ & $\begin{array}{l}\text { Broad activity window } \\
\text { Circularly permuted SpCas9 }\end{array}$ & {$[104]$} \\
\hline CBE-NRRH, CBE-NRCH, CBE-NRTH & \multicolumn{2}{|c|}{ As BE4max } & $(3) 4-8$ & $\begin{array}{l}\text { Evolved non-G PAMs are NRRH, } \\
\text { NRCH(\&NGH), NRTH }\end{array}$ & {$[105]$} \\
\hline $\begin{array}{l}\text { SpG-CBE, SpRY-CBE (produced by the } \\
\text { Kleinstiver lab) }\end{array}$ & \multicolumn{2}{|c|}{ As BE4max } & $(3) 4-8$ & $\begin{array}{l}\text { PAMs are NGH, NRN }>\text { NYN } \\
\text { SpRY relaxed PAM preference } \\
\text { Available as HF1 high fidelity }\end{array}$ & {$[106]$} \\
\hline
\end{tabular}


Table 4. List of adenine base editors. Chronological production of ABEs by David R. Liu's lab.

\begin{tabular}{|c|c|c|c|c|c|}
\hline \multicolumn{2}{|l|}{ Adenine base editors } & \multicolumn{4}{|c|}{ Abbreviation: arch', architecture; evo', evolution/evolved } \\
\hline $\begin{array}{l}\text { Adenine base editor name / } \\
\text { Architecture }\end{array}$ & NLS & \begin{tabular}{|l|} 
Codon \\
optimize
\end{tabular} & $\begin{array}{l}\text { Activity } \\
\text { window }\end{array}$ & Features & Ref \\
\hline $\begin{array}{l}\text { ABE6.3 } \\
\text { wtTadA-32aa-TadA*-32aa-Cas9(D10A)-NLS }\end{array}$ & SV40 & No & $4-9$ & 6th evolved ABE & {$[37]$} \\
\hline $\begin{array}{l}\text { RA6.3 } \\
\text { FLAG-NLS-wtTadA-32aa-TadA*-32aa-Cas9(D10A)-NLS }\end{array}$ & $\begin{array}{l}2 \mathrm{xSV}- \\
40\end{array}$ & $\begin{array}{l}\text { Yes-Cas- } \\
\text { 9n RA }\end{array}$ & $4-9$ & $\begin{array}{l}\text { Improved protein expression and } \\
\text { localization in mammalian cells }\end{array}$ & $\begin{array}{l}{[56,1} \\
07]\end{array}$ \\
\hline $\begin{array}{l}\text { ABE7.10 } \\
\text { wtTadA-32aa-TadA*-32aa-Cas9(D10A)-NLS }\end{array}$ & SV40 & No & $4-7$ & 7th evolved ABE & {$[37]$} \\
\hline $\begin{array}{l}\text { ABEmax } \\
\text { bpNLS-wtTadA-32aa-TadA*-32aa-Cas9(D10A)-bpNLS }\end{array}$ & $\begin{array}{l}2 \text { xbi- } \\
\text { partite }\end{array}$ & $\begin{array}{l}\text { Yes-Ge- } \\
\text { nScript }\end{array}$ & $4-7$ & $\begin{array}{l}\text { 7th evolved ABE with max arch' } \\
\text { Improved protein expression and } \\
\text { localization in mammalian cells }\end{array}$ & {$[100]$} \\
\hline $\begin{array}{l}\text { SpABE8e } \\
\text { bpNLS-TadA*-32aa-Cas9(D10A)-bpNLS }\end{array}$ & $\begin{array}{l}2 \text { xbi- } \\
\text { partite }\end{array}$ & $\begin{array}{l}\text { Yes-Ge- } \\
\text { nScript }\end{array}$ & $4-8$ & $\begin{array}{l}\text { 8th evolved ABE monomeric } \\
\text { construct, increased processivity } \\
\text { Phage-assisted continuous evo' }\end{array}$ & {$[41]$} \\
\hline $\begin{array}{l}\text { SpABE8e V106W } \\
\text { bpNLS-TadA*(V106W)-32aa-Cas9(D10A)-bpNLS }\end{array}$ & $\begin{array}{l}\text { 2xbi- } \\
\text { partite }\end{array}$ & $\begin{array}{l}\text { Yes-Ge- } \\
\text { nScript }\end{array}$ & $4-8$ & $\begin{array}{l}\text { Decreased TadA DNA/RNA } \\
\text { affinity, reduced off-target Cas9- } \\
\text { independent base conversion }\end{array}$ & {$[41]$} \\
\hline \multicolumn{6}{|c|}{ Adenine base editors with altered PAM specificity or activity window size } \\
\hline xCas9-ABE7.10 & \multicolumn{2}{|c|}{ As ABE7.10 } & $4-7$ & $\begin{array}{l}\text { Successful PAMs are NGG/NGA/ } \\
\text { NGC/GAT(NGNC), evo' SpCas9 }\end{array}$ & {$[102]$} \\
\hline SaABEmax, SaKKH-ABEmax & \multicolumn{2}{|c|}{ As ABEmax } & $\begin{array}{l}4-14 \\
\text { single }\end{array}$ & PAMs are NNGRRT, NNNRRT & {$[104]$} \\
\hline $\begin{array}{l}\text { VRQR-ABEmax, VRER-ABEmax, } \\
\text { xABEmax, NG-ABEmax }\end{array}$ & \multicolumn{2}{|c|}{ As ABEmax } & $4-7$ & $\begin{array}{l}\text { PAMs are NGA/NGCG/NGCC, } \\
\text { NGCG, NGCC/GAT(NGNC), } \\
\text { NGCG/NGCC/GAT(NGH) } \\
\text { respectively } \\
\text { Optimal base editor may vary on } \\
\text { a site by site basis }\end{array}$ & [104] \\
\hline SaABE8e, SaKKH-ABE8e & \multicolumn{2}{|c|}{ As SpABE8e } & $3-14$ & PAMs are NNGRRT, NNNRRT & [41] \\
\hline dLbCas12a-ABE8e, enAsCas12a-ABE8e & \multicolumn{2}{|c|}{ As SpABE8e } & $8-14$ & $\begin{array}{l}\text { PAMs are TTTV, } \\
\text { TTYN/VTTV/TRTV } \\
\text { Dead LbCas12a, engineered } \\
\text { AsCas12a }\end{array}$ & $\begin{array}{l}{[41,1} \\
08]\end{array}$ \\
\hline NG-ABE8e & \multicolumn{2}{|c|}{ As SpABE8e } & $4-8$ & $\begin{array}{l}\text { PAM is NGH (lower activity on } \\
\text { PAM NGC) }\end{array}$ & $\begin{array}{l}{[41,1} \\
03]\end{array}$ \\
\hline $\begin{array}{l}\text { CP1012-ABEmax, also CP1028-, CP1041-, } \\
\text { CP1249- }\end{array}$ & \multicolumn{2}{|c|}{ As ABEmax } & $\begin{array}{l}4-12 \\
1041: 14\end{array}$ & $\begin{array}{l}\text { Broad activity window } \\
\text { Circularly permuted SpCas9 }\end{array}$ & [104] \\
\hline СР1028-ABE8e, CР1041- & \multicolumn{2}{|c|}{ As SpABE8e } & $3-14$ & $\begin{array}{l}\text { Broad activity window } \\
\text { Circularly permuted SpCas9 }\end{array}$ & {$[41]$} \\
\hline ABE-NRRH, ABE-NRCH, ABE-NRTH & \multicolumn{2}{|c|}{ As ABEmax } & $4-7$ & $\begin{array}{l}\text { Evolved non-G PAMs are NRRH, } \\
\text { NRCH(\&NGH), NRTH }\end{array}$ & [105] \\
\hline $\begin{array}{l}\text { SpG-ABE, SpRY-ABE (produced by the } \\
\text { Kleinstiver lab) }\end{array}$ & \multicolumn{2}{|c|}{ As ABEmax } & $4-7$ & $\begin{array}{l}\text { PAMs are NGH, NRN }>\text { NYN } \\
\text { SpRY relaxed PAM preference } \\
\text { Available as HF1 high fidelity }\end{array}$ & [106] \\
\hline
\end{tabular}




\section{CRISPR/base editing in Xenopus embryos.}

For injection into newly fertilized embryos, the properties of base editors seem particularly suited to species with longer maturation times. The indel variability and mosaicism that would otherwise result from repair of Cas9 DSBs can be avoided. Providing the deaminase can contact all copies of the targeted gene, uniformly homozygous animals carrying defined mutations may be obtained among G0-generation cohorts. Indeed, following injection of pronuclear stage rabbit zygotes to target several genes, precise mutations were recovered in $53-88 \%$ and $44-100 \%$ of growing blastocysts for a CBE and ABE respectively, with 100\% allele conversion and no evidence of mosaicism within the successfully adenine edited embryos [60].

Two studies have so far reported the efficiency of base editing in Xenopus embryos, both with the CBE, BE3. An early attempt in $X$. laevis with a lab preparation of BE3 protein produced a modest $20 \%$ base conversion that introduced a premature stop codon into both homeologous copies of the tyr gene (albino phenotype), though also accompanied by a 15\% rate of unwanted indels and mosaicism [51]. In $X$. tropicalis, 10 genes were examined with an emphasis on reproducing human disease causing mutations. Of 19 gRNAs tested with BE3 mRNA, 11 gave base edits with efficiencies $>15 \%$, with a $t b x 5$ mutation encoding the R237W substitution occurring in $36 \%$ of examined sequences [18]. The newer, evolved versions of the base editors represent a golden opportunity for Xenopus researchers to generate homozygous point mutations routinely in G0embryos. As a priority, they must be tested to measure what base conversion efficiencies can be produced. Even if their activity is deemed too low to achieve homozygous installation, then the absence of allele variability (and few off-target effects) still means that clean recessive phenotypes can be analysed by rearing and then mating the resulting frogs.

Base editors offer a number of different experimental strategies. Specific missense mutations can be placed within genes for study of their effects (Fig 1), or as an alternative, the production of nonsense mutations can be targeted that precisely truncate the translated protein product (Fig 2). Termination codons are introduced by CBEs; CAA, CAG and CGA triplets mutate to TAA, TAG and TGA with sense oriented gRNAs, while TGG converts to all three stop codons using antisense gRNAs. One further point is how the choice of base editors and PAM sequences has brought new flexibility to gRNA design. Previously, CRISPR projects began with a search of your gene of interest to locate regions that could be targeted by a gRNA, which was constrained by rigid sequence requirements. Now the initial search is fundamentally different; to identify the precise sequence changes wanted in your gene and then to select the base editor variant and gRNA position that will perform the task. And if no suitable options are available, then consider a prime editor strategy instead. 


\section{Fig 1. Base editing example: ABE arginine substitution in the Adprhl1 substrate binding cleft}

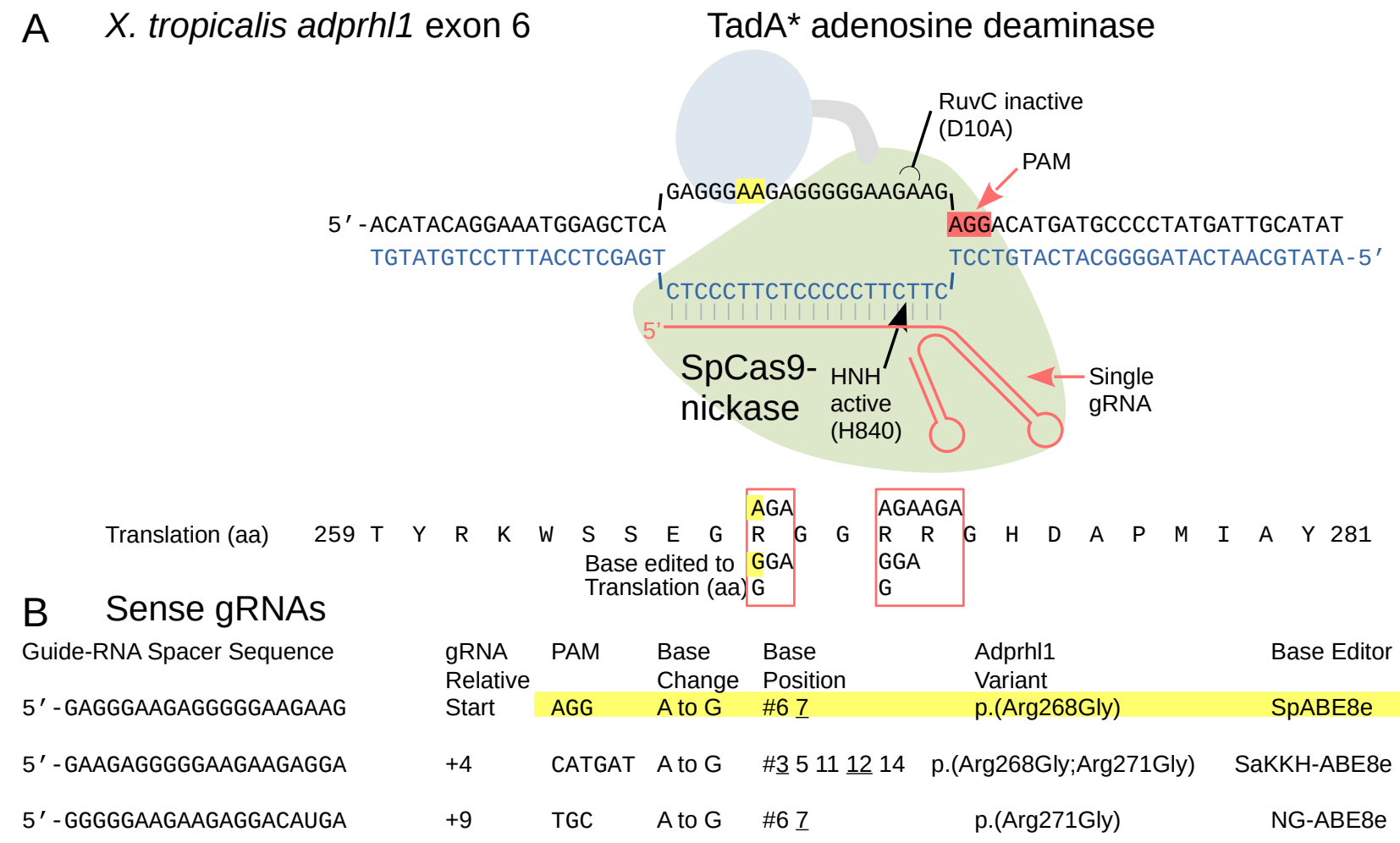

A: Part of the $X$. tropicalis adprhl1 gene exon 6 showing hybridization of a single-gRNA to its target sequence. The complex of the gRNA with an ABE illustrates the region of the displaced DNA strand that is accessible to the TadA* adenosine deaminase, with editable bases highlighted. The Cas9-nickase HNH nuclease domain of the ABE cuts only the target DNA strand that hybridizes with the gRNA. The Adprhl1 amino acid sequence is shown underneath.

Adprhl1is a fascinating pseudoenzyme that is required for muscle growth within the embryonic heart, although its mechanism of action has not yet been resolved. When complexed with Cas9 nuclease, this gRNA produces a strong cardiac phenotype in CRISPant embryos associated with a number of small in-frame deletions that eliminate arginine residues from a peptide loop at the centre of the ancestral catalytic active site (marked by red boxes) [14]. By utilizing adenine base editing, a functional analysis of individual arginines is now made possible.

B: Table showing the projected results of ABE experiments in this region. The top line represents the illustrated gRNA whose base conversion substitutes arginine-268 to glycine. By selecting different sense gRNA sequences, moved 4 and 9 bases towards the right, and using ABE variants with alternate PAM requirements, substitution of arginine-271 can also be achieved. The codon for arginine-272, conversely, is more challenging to mutate. It can be reached by a base editor with a relaxed PAM preference (SpRY-ABE, gRNA moved 15 bases to the right, using TAT as a PAM) or by adopting a prime editor strategy (Fig 3). This represents a typical scenario for a reverse genetics project, where base editors and prime editors introduce most of the exploratory mutations required to dissect gene and protein function. Only larger DNA sequence insertions need invoke Cas9 DSB and HDR-mediated mechanisms. For Adprhl1, base editing and prime editing will reveal whether the arginines participate directly in cardiac myofibril assembly or if preservation of the peptide loop size and structure is all that is necessary. 


\section{Fig 2. Base editing example: CBE nonsense mutations to truncate the Adprhl1 protein}

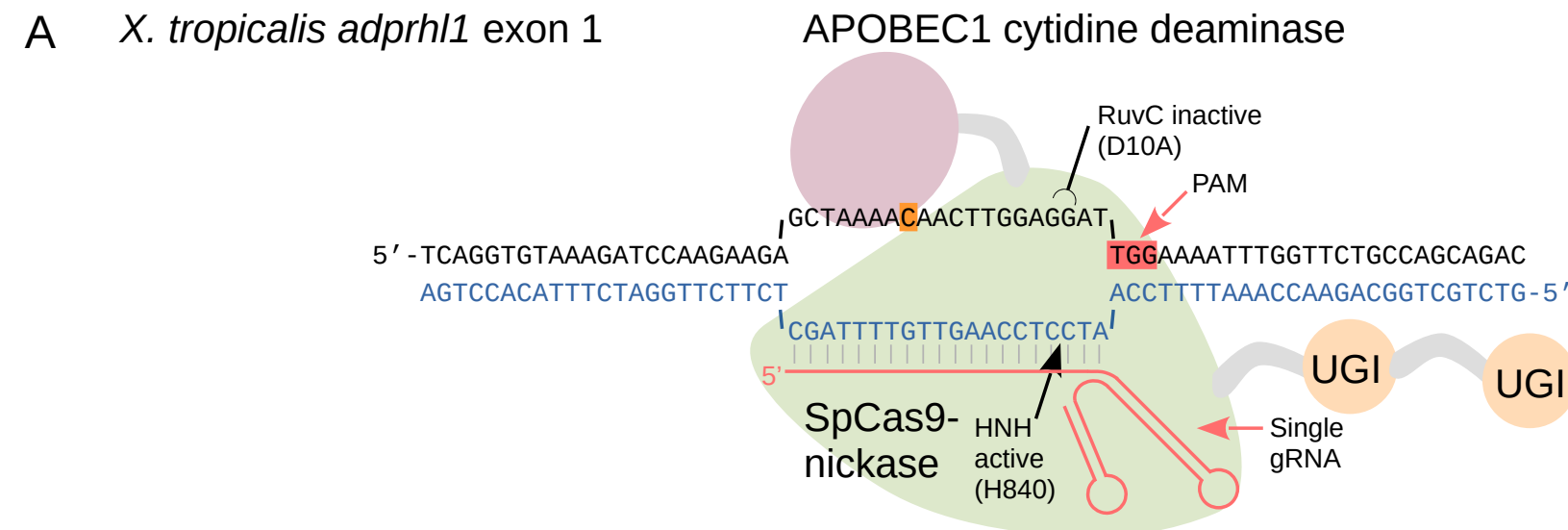

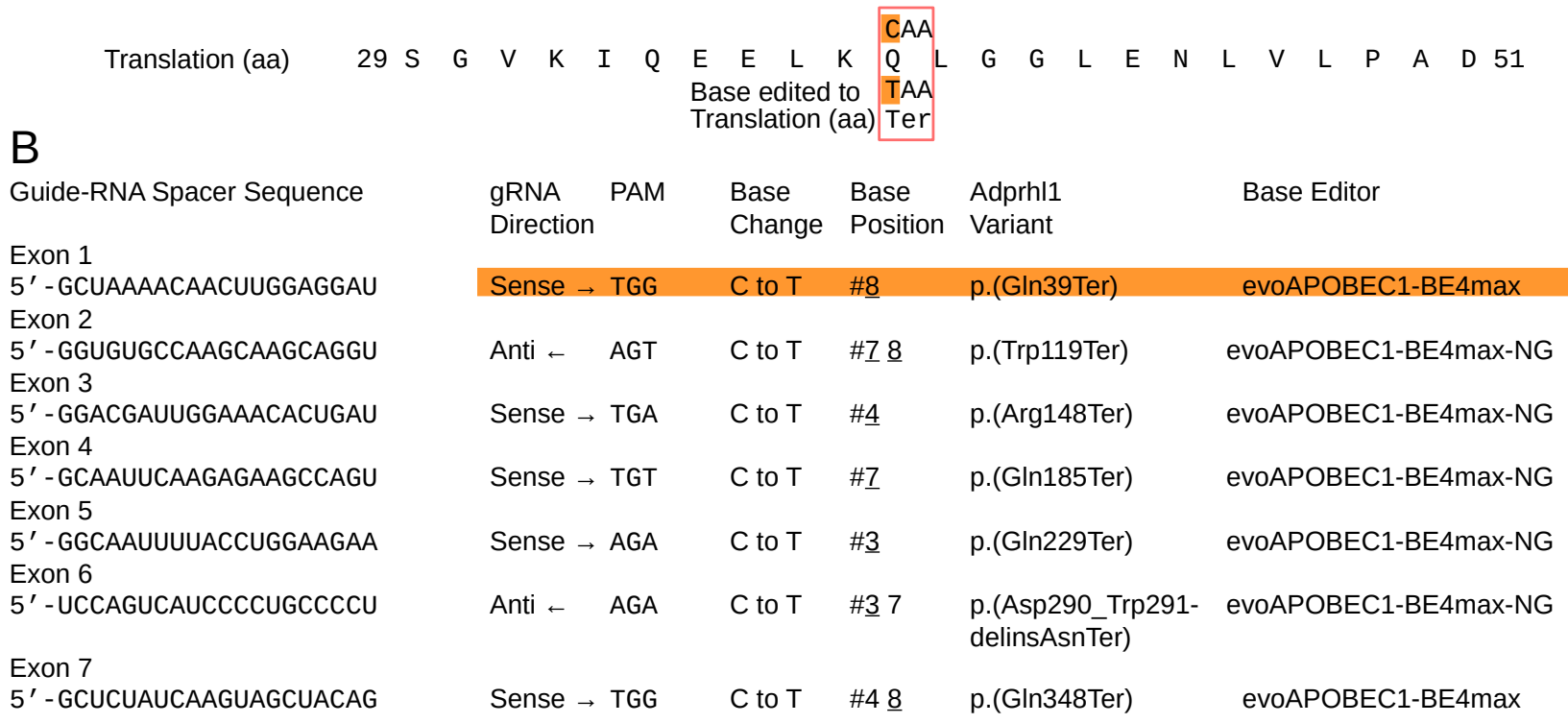

A: Part of the $X$. tropicalis adprhl1 gene exon 1 showing hybridization of a single-gRNA to its target sequence. The complex of the gRNA with a CBE illustrates the region of the displaced DNA strand that is accessible to the APOBEC1 cytidine deaminase, with an editable base highlighted. The relevant Adprhl1 amino acid sequence is shown underneath. Base editing converts a glutamine codon into a chain termination TAA (ochre) codon (red box).

B: List of gRNAs and CBEs capable of introducing nonsense mutations into each exon of the adprhl1 gene. A series of stop codons like this can be produced rapidly for most genes and offers greater precision compared to the alternative strategy of generating frame-shift mutations with Cas9 DSB repair. The approach is useful for exploring the contribution of exons to different protein isoforms for a gene with complex patterns of RNA-splicing. 


\section{CRISPR/prime editing for transversion mutations, accurate small insertions and deletions.}

Prime editors offer the greatest versatility for precise installation of a wide range of genetic sequence changes [61] and will likely see increasing use in Xenopus and zebrafish embryo research. They consist of the H840A nickase version of Cas9, with a C-terminal fusion via a linker to a reverse transcriptase enzyme. In this configuration, the active RuvC nuclease domain (D10) nicks only the displaced DNA strand (the PAM-strand) at the targeted sequence. The second key component is a longer, prime editing guide RNA (pegRNA). Its 5'-sequence (the spacer sequence) hybridizes the target DNA strand as usual, but additional bases placed at the 3 '-end can reach across to pair with the displaced strand (Fig 3A). The 3'-hydroxyl at the DNA nick, combined with the base paired RNA, acts as a priming site for nucleotide polymerization by the reverse transcriptase that can be utilized to replace part of the displaced strand DNA with new sequence information.

Reading in from the 3'-end, the pegRNA requires 8-15 bases complementary to the displaced strand that will hybridize 5'-of the DNA nick position, which is termed the primer binding site (PBS). This is followed by the reverse transcriptase template (RTT) region where revised sequence can be incorporated [61]. The immediate product of RTT nucleotide extension is a branched DNA intermediate with an overhanging 3'-flap on the displaced strand containing edited sequence (Fig 3B). Competition for base pairing to the target strand between this extension and the original unedited bases then leads to an equilibrium between the 3'-flap or alternately an exposed 5'-flap. While hybridization of the perfectly complementary unedited bases should be favoured, any exposed 5'-flap would be a preferred substrate for endonucleases such as FEN1 [62] and also 5'exonucleases like EXO1 [63]. The susceptibility to degradation of 5'-overhangs thus drives the incorporation and subsequent ligation of the edited sequence into the displaced strand (Fig 3C).

Optimization of this recently developed technology in cultured cells is at an early stage compared to base editors. It has included testing both $\mathrm{N}$ - and C-terminal fusions of Cas9 nickase and reverse transcriptase, plus alternate pegRNA configurations with the PBS at the 5' versus 3'-end. Two active prime editors were initially characterized [61]. The first (PE1) used wild-type Moloney murine leukemia virus (M-MLV) reverse transcriptase. The second (PE2) included five established amino acid variants that enhance reverse transcriptase performance (Table 5). Strengthening the NLS has yielded an improved PE2* variant [64] and the first prime editors that offer a choice of different PAM sequences have now been constructed $[64,65]$.

To complete the process and maximize efficiency, the non-edited DNA strand, that is the strand targeted by the pegRNA spacer sequence, should also be converted to complement the newly edited displaced strand. Similar to base editing, nicking the non-edited strand is again utilized to encourage the mismatch repair pathway. But because the displaced strand is also nicked to initiate prime editing, different strategies are needed in order to avoid DSB formation and consequent indels. One approach, termed PE3 [61], is to add another simple gRNA that hybridizes in the opposite orientation and targets the H840A nickase to a distinct site at a distance from the editing position (known as a nicking gRNA, ngRNA). A non-edited strand nick, placed 40-90 bp 3'-from the pegRNA-induced nick (counted on the displaced strand, Fig 3D) generally improves editing outcomes without increasing unwanted indel frequency. A more elegant solution, termed PE3b [61], is to design the spacer sequence of the second ngRNA to hybridize the newly edited bases of the displaced strand. This sequential method ensures the second nick will only occur once the edited strand repair has ligated, but does depend on the proximity of a suitable PAM for the ngRNA. One further design consideration is to make sure that the pegRNA, and optimally also the ngRNA, will not act on the revised DNA sequence once prime editing and mismatch repair have completed. In a PE3b strategy, this could entail synonymous mutation of bases complementary to the second PAM during RTT extension on the displaced strand, so that this PAM is disrupted after mismatch repair. 
The strength of prime editing lies in the great variety of genome edits that can be produced. All 12 types of single base changes are possible, including the transversion mutations where a pyrimidine nucleotide is switched to a purine, or vice versa. Two other potential limitations of base editors are also resolved by prime editing. Bystander mutations can be problematic for base editors, in examples where a targeted site contains multiple editable bases within the activity window yet it is paramount that only a single one is converted. For a prime editor, such replacement of a particular base is routine as the exact sequence to be installed is specified by the RTT region of the pegRNA. Prime editors additionally allow greater flexibility with regard to the distance of the desired edit to a suitable PAM for the pegRNA (Fig 3E).

Transversion mutation efficiencies reported for multiple genomic loci in cultured cells have commonly ranged between $25-40 \%$; upwards of 55\% being possible [61]. The highest values occurred when the edited nucleotide disrupted the PAM used by the pegRNA, at base +5 or +6 from the pegRNA-induced nick position, to prevent further nicking of the edited strand prior to mismatch repair of the complementary strand. Similar conversion rates could be obtained for long-range transversions placed up to 33 bases from the pegRNA-nick using extended RTT sequences as well as adjacent gene edits installed with short RTTs. Precise DNA insertions of 1 to $3 \mathrm{bp}$, or deletions of 1 to $3 \mathrm{bp}$, targeted between positions +1 to +6 also achieved efficiencies of 29-39\%. Moreover, insertion of epitope tags, larger deletions up to $80 \mathrm{bp}$ with accurately specified boundaries, plus combinations of different mutations were all successfully produced [61]. The main disadvantage, in addition to the lower overall activity, is the frequency of non-specified indels at a prime edited site, which is currently greater than that caused by base editors. They occurred with a frequency of 5-8\% across the different PE3 experiments (occasionally 20\%), while the improved suppression of DSBs by a PE3b strategy was able to reduce indels to $0.74 \%$ [61].

The prime editing mechanism can enhance genomic target specificity because of the additional requirement for pegRNA complementarity extending through the RTT region. This is needed to ensure that the synthesized DNA 3'-flap of the edited strand is able to hybridize to the non-edited strand and promotes integration solely at the correct target. Consequently, DNA edits at potential off-target sites defined by spacer sequence similarity occur very rarely using PE2/PE3 and pegRNAs $(<0.1 \%$ frequency) [61]. Further unbiased sequencing methods that revealed all off-target positions across the genome where a guided H840A-nuclease would nick purified DNA in vitro, in most cases, did not identify any sites where the corresponding PE2-pegRNA complex acting in cells caused mutations [66]. Even genome-wide sequencing of PE-treated plants has found no evidence for off-target genome alteration by prime editors [67].

The assessment of prime editing for genome manipulation tasks in stem cells $[68,69]$ and laboratory model organisms, as well as appraisal of its biotech' and therapeutic potential, is currently underway. In mice, PE3 injection of embryos (PE2-encoding plasmid, pegRNA and ngRNA) successfully introduced two single nucleotide pathogenic transversions ( $<18.5 \%$ efficiency) with no off-target edits, albeit accompanied by some mosaicism with other conversions of the targeted base [70]. PE2 mRNA injection produced a cleaner installation of a specific transcription factor binding site transversion mutation ( $<51 \%$ efficiency, mean $21 \%$ ) without any contaminating on-target indels [71]. Adult mice have also received prime editors through systemic administration of plasmid and via dual adeno-associated viruses [64]. Elsewhere, prime editors have proved similarly effective for precise editing of Drosophila genes [72]. Software has already been developed to automate design of the complex pegRNA and second ngRNA components. They simplify the identification of PE3b guide combinations $[73,74]$ and incorporation of synonymous mutations for ngRNA PAM disruption [73]. It is hoped that ongoing development of prime editors will follow a similar path to 
that achieved by base editors, with rapid improvements in activity, maximum insertion size and ontarget precision becoming available. Base editor and prime editor systems complement each other and enable many genome engineering projects, including those in Xenopus embryos, to be accomplished without resorting to DSB repair mechanisms.

Table 5. List of prime editors. Chronological production of PEs with recommended pegRNA and ngRNA design parameters.

\begin{tabular}{|c|c|c|c|c|c|}
\hline \multicolumn{3}{|l|}{ Prime editors } & \multicolumn{3}{|c|}{ Abbreviation: arch', architecture } \\
\hline \multicolumn{2}{|l|}{$\begin{array}{l}\text { Prime editor name / } \\
\text { Architecture }\end{array}$} & NLS & \multicolumn{2}{|l|}{ Features } & Ref \\
\hline \multicolumn{2}{|l|}{$\begin{array}{l}\text { PE1 } \\
\text { bpNLS-Cas9(H840A)-33aa-M-MLVRT-bpNLS }\end{array}$} & $\begin{array}{l}\text { 2xbi- } \\
\text { partite }\end{array}$ & \multicolumn{2}{|c|}{ Original prime editor } & {$[61]$} \\
\hline \multicolumn{2}{|l|}{$\begin{array}{l}\text { PE2 } \\
\text { bpNLS-Cas9(H840A)-33aa-M-MLVRT*-bpNLS }\end{array}$} & $\begin{array}{l}\text { 2xbi- } \\
\text { partite }\end{array}$ & \multicolumn{2}{|c|}{$\begin{array}{l}\text { Pentamutant M-MLV reverse transcriptase } \\
\text { D200N, T330P, L603W support reverse transcription } \\
\text { at elevated incubation temperatures } \\
\text { T306K, W313F enhance priming site binding, enzyme } \\
\text { processivity and thermostability }\end{array}$} & {$[61]$} \\
\hline \multicolumn{2}{|l|}{ PE3, PE3b } & $\begin{array}{l}\text { As } \\
\text { PE2 }\end{array}$ & \multicolumn{2}{|c|}{$\begin{array}{l}\text { Strategy uses PE2, pegRNA plus additional ngRNA to } \\
\text { ensure conversion of non-edited DNA strand (below), } \\
\text { at the expense of higher on-target indel rates for PE3 }\end{array}$} & {$[61]$} \\
\hline \multicolumn{2}{|c|}{$\begin{array}{l}\text { PE2* } \\
\text { C-MycNLS-bpNLS-Cas9(H840A)-33aa-M-MLVRT*- } \\
\text { vbpNLS-SV40NLS }\end{array}$} & $\begin{array}{l}4 \mathrm{x} \\
\mathrm{NLS}\end{array}$ & \multicolumn{2}{|c|}{$\begin{array}{l}\text { Strengthened NLS } \\
\text { N-term has C-Myc NLS and bipartite (SV40) NLS } \\
\text { C-term has variant bpNLS and SV40 NLS }\end{array}$} & {$[64]$} \\
\hline \multicolumn{6}{|c|}{ Prime editors with altered PAM specificity } \\
\hline \multicolumn{2}{|c|}{$\begin{array}{l}\text { SaPE2*, Sa }{ }^{\mathrm{KKH}} \mathrm{PE2}{ }^{*} \\
\text { C-MycNLS-bpNLS-SaCas9(N580A)-33aa-M-MLVRT*- } \\
\text { vbpNLS-SV40NLS }\end{array}$} & $\begin{array}{l}\text { As } \\
\text { PE2* }\end{array}$ & \multicolumn{2}{|c|}{$\begin{array}{l}\text { SaCas9 nickases with PE2* architecture } \\
\text { PAMs are NNGRRT, NNNRRT }\end{array}$} & {$[64]$} \\
\hline \multicolumn{2}{|l|}{ PE2-VQR, PE2-VRQR } & $\begin{array}{l}\text { As } \\
\text { PE2 }\end{array}$ & \multicolumn{2}{|c|}{$\begin{array}{l}\text { Engineered SpCas9 nickases with PE2 arch' } \\
\text { PAM is NGA for both }\end{array}$} & {$[65]$} \\
\hline \multicolumn{2}{|l|}{ PE2-VRER } & PE2 & \multicolumn{2}{|c|}{ PAM is NGCG } & [65] \\
\hline \multicolumn{2}{|l|}{ PE2-NG, PE2-SpG } & PE2 & \multicolumn{2}{|c|}{ PAM is NGH for both } & {$[65]$} \\
\hline \multicolumn{2}{|l|}{ PE2-SpRY } & PE2 & \multicolumn{2}{|c|}{ Relaxed PAM is NRN $>\mathrm{NYN}$} & {$[65]$} \\
\hline pegRNA design parameters & \begin{tabular}{|l|} 
PBS \\
length
\end{tabular} & \begin{tabular}{l|l} 
RTT & F \\
length & ( \\
\end{tabular} & $\begin{array}{l}\text { Position of edit } \\
\text { (post-nick) }\end{array}$ & Additional guidelines & Ref \\
\hline $\begin{array}{l}\text { Recommended parameters for initial } \\
\text { design of pegRNAs (some } \\
\text { optimization will be needed) }\end{array}$ & 13 & $\begin{array}{l}10-16 \\
(<34)\end{array}$ & $\begin{array}{l}+1 \text { to }+6 \\
+5,+6 \text { disrupts } \\
\text { pegRNA PAM }\end{array}$ & $\begin{array}{l}\text { Avoid C-base at first position of the } \\
\text { RNA 3'-extension } \\
\text { Minimum number of complementary } \\
\text { bases after the edit on the DNA 3'-flap } \\
\text { should be 5, optimally } 10\end{array}$ & $\begin{array}{l}{[61,7} \\
3,74]\end{array}$ \\
\hline ngRNA design parameters & \multicolumn{4}{|c|}{ Guidelines } & \\
\hline PE3 strategy & \multicolumn{4}{|c|}{$\begin{array}{l}\text { Opposite orientation to pegRNA } \\
\text { ngRNA nick placed 40-90 bp (3'-) from pegRNA nick }\end{array}$} & {$[61]$} \\
\hline PE3b strategy & \multicolumn{4}{|c|}{$\begin{array}{l}\text { Opposite orientation to pegRNA } \\
\text { ngRNA spacer hybridizes to edited displaced strand } \\
\text { Optimally, ngRNA PAM position overlaps the complementary sequence } \\
\text { between the pegRNA nick and pegRNA PAM, allows PAM disruption }\end{array}$} & $\begin{array}{l}{[61,7} \\
3,74]\end{array}$ \\
\hline
\end{tabular}


Fig 3. Prime editing example: replacement of arginine-272 in the Adprhl1 substrate binding cleft

\section{A X. tropicalis adprhl1 exon $6 \quad$ M-MLV reverse transcriptase effector}

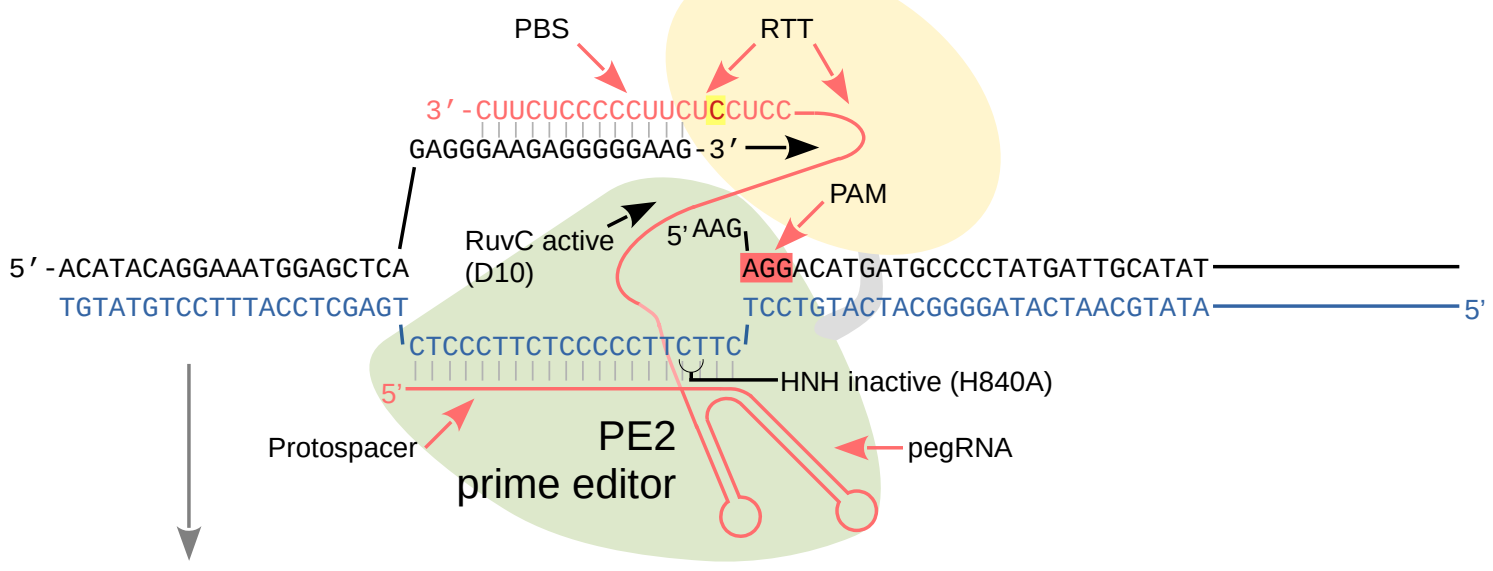

B 3'-Flap resolution of the edited DNA strand ${ }_{A G G A G A C A T G A T G C C}-3^{\prime}$

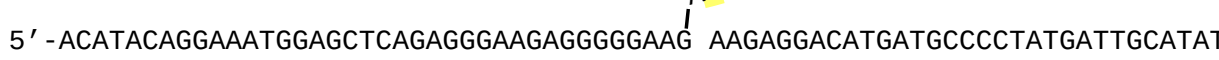

TGTATGTCCTTTACCTCGAGTCTCCCTTCTCCCCCTTC -TTCTCCTGTACTACGGGGATACTAACGTATA

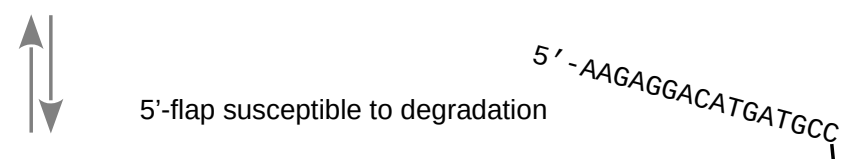

5' - ACATACAgGAAATGgAGCTCAgAgGgAAgAgGgGgAagAGgAgGACATGATGCC CCTATGATTGCATAT

TGTATGTCCTTTACCTCGAGTCTCCCTTCTCCCCCTTCT TCTCCTGTACTACGG - GGATACTAACGTATA

$\checkmark$

C Ligation of the edited DNA strand

5' - ACATACAGGAAATGGAGCTCAGAGGGAAGAGGGGGAAGAGGAGGACATGATGCCCCTATGATTGCATAT

TGTATGTCCTTTACCTCGAGTCTCCCTTCTCCCCCTTCT TCTCCTGTACTACGGGGATACTAACGTATA

$\checkmark$

D PE3: distant nick-mediated mismatch repair of the non-edited (target) DNA strand

ngRNA $\rightarrow$

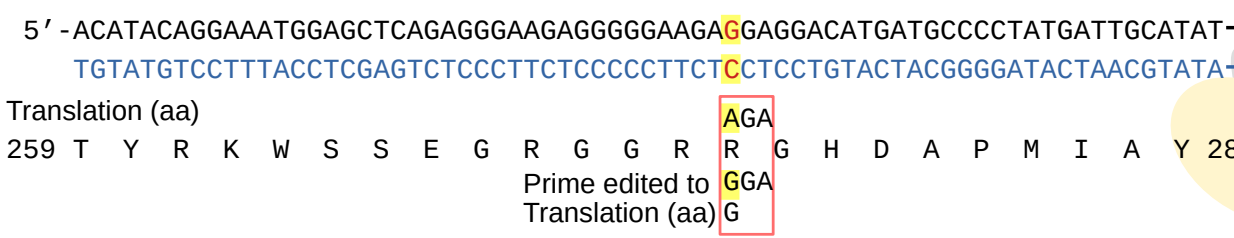

\section{E pegRNA and ngRNA sequences}

pegRNA Spacer Sequence Direction PAM

5'-GAGGGAAGAGGGGAAGAAG $\rightarrow$ AGG

Additional mut' to disrupt pegRNA PAM

5' - UGGAGCUCAGAGGGAAGAGG

PE3 ngRNA Spacer Sequence

5 ' - GUAUGUCUGUUGGAUAGGCG

$5^{\prime}$ - GAGCAUCAUAUGCAAUCAUA
PAM

GGG

GGG
pegRNA RTT

pegRNA PBS

$5^{\prime}$-ggCAUCAUGUCCUCCUCUUCCCCCUCUUC

$5^{\prime}$ - GGCAUCAUGUGCUCCUCUUCCCCCUCUUC

GGG 5'-UCAUGUCCUCCUCCUCCCCCUCUUCCCUCUGAGC

p.(Arg272Gly)

p.(Arg272_Gly273delinsGlyAla)

p.(Arg271_Arg272delinsGlyGly)
(pegRNA nick to ngRNA nick)

$-49$

+21 (additional ngRNA choices at $+149,+174,+194$ ) 


\section{Fig 3. Prime editing example: replacement of arginine-272 in the Adprhl1 substrate binding cleft}

A: Part of the $X$. tropicalis adprhl1 gene exon 6 with hybridization of a pegRNA to its target sequence. The complex of the pegRNA with PE2 nicks the displaced DNA strand and primes nucleotide polymerization by the reverse transcriptase. The presence of a point mutation within the RTT is highlighted. The protospacer of the pegRNA is the same as that featured in the Fig 1 base editing example. B: DNA extension results in an overhanging 3'-flap on the displaced strand containing edited sequence that competes with the original bases for pairing to the target strand. A hyphen (-) has been placed within the non-edited target strand sequence to simplify drawing of the 3'-flap versus alternate 5'-flap arrangements. C: Increased degradation of any exposed 5'-overhang aids incorporation of the edit into the displaced strand. D: Hybridization of a second ngRNA in the opposite orientation promotes conversion of the nonedited strand through mismatch repair. To avoid DSBs, the ngRNA spacer is sited distant to the pegRNA (this, PE3 strategy) or selectively hybridizes to the edited sequence (PE3b strategy). The Adprhl1 amino acid sequence is shown underneath.

E: Prime editors can introduce any transition or transversion mutation, plus defined insertions and deletions. Table lists three pegRNAs that target this adprhl1 region, with columns for 5'-spacer sequences, plus RTT and PBS 3'-extensions. The top line pegRNA directs a single transition that cannot be reached by conventional base editors, which substitutes arginine-272 to glycine. The second pegRNA adds a transversion to disrupt the pegRNA PAM and hence increase editing efficiency, which in this case also switches glycine-273 to alanine. The third pegRNA spacer is moved 9 bases towards the left and its RTT incorporates two transitions that replace both vicinal arginines-271 and -272. Two ngRNAs for PE3 conversion of the non-edited strand are also listed. Together, the base editor and prime editor exon 6 examples provide for a complete exploration of arginine dependency in the Adprhl1 pseudoenzyme active site.

\section{Methods to save a generation during Xenopus gene mutation studies.}

The traditional strengths of Xenopus embryo experiments are that they are rapid, performed at large scale and for low cost. But as discussed previously, reverse genetic approaches that require transmission of mutations through successive generations of frogs don't easily fit this ethos. Hence, in addition to base editing and prime editing, other Xenopus-specific solutions to speed up production of homozygous, Cas9 nuclease DSB-induced mutants have been developed.

Leapfrogging is a term given to transfer of primordial germ cells (PGC) from a Cas9-gRNA injected donor embryo into a PGC-depleted wild-type host embryo [26]. The procedure is a straightforward surgical transplantation of the vegetal pole region at the post-mid-blastula transition stage [75]. It has permitted PGCs with efficiently mutated genomes to be raised in healthy animals with normal somatic cells. Interbreeding of leapfrog hosts then produced embryos where the majority carried compound heterozygous mutations of the targeted gene, allowing knock-out phenotype analysis at the F1-generation. The technique has several advantages. The injection and grafting procedure per se does not affect the embryo phenotype. Genes that function in the early embryo via maternally synthesized mRNA can be studied, which is not possible by mutation and immediate G0-generation analysis. Significantly, there is no cellular mosaicism of mutated alleles so any developmental defect observed within tissues of an individual F1-embryo will be homogeneous. The only downside is that the variability of mutations that arise from different DSB repairs will still persist among the cohort of examined embryos.

A second technique that yields non-mosaic gene mutation is to induce the DSBs in Xenopus oocytes. This somewhat laborious procedure has been optimized for both $X$. laevis and $X$. tropicalis $[76,77]$. Oocytes were isolated from ovarian tissue, incubated with Cas9-gRNA reagents, then matured before transferral into an ovulating host frog for subsequent in vitro fertilization (termed oocyte host-transfer). Although only a portion (11\%) of injected oocytes successfully developed into embryos, some $90 \%$ of the resulting G0-tadpoles were heterozygous, carrying a single indel in their maternally inherited copy of the gene. By raising these heterozygotes, genotyping their mutations and interbreeding, Mendelian ratios of true homozygous mutant F1-embryos can be recovered (or uniform compound heterozygotes). 


\section{DSB repair options for larger DNA sequence insertions in Xenopus embryos.}

A Cas9-guided DSB can be used as an entry point to target the insertion of new DNA sequences into a gene. The attraction, compared to current prime editors, is that longer insertions are possible. In cell culture systems, a means of positive cell selection is usually incorporated alongside the desired insertion (either by a drug selection construct or by cell sorting). But when introducing novel sequences into embryos, without a selection step, it may be necessary to screen many potential founders for the presence of rare, and probably mosaic, DNA integration events. The challenge, therefore, is to increase their frequency of occurrence, while maintaining precise boundaries between the endogenous and inserted sequence.

Many articles have explained how homology directed repair (HDR) pathways can be co-opted for gene editing purposes (see https://blog.addgene.org/crispr-101-homology-directed-repair) [78]. Insertion depends upon supplying a donor template, which contains the new sequence, flanked by homology arms that match the sequence surrounding the cleavage site and allow for homologous recombination of complementary DNA strands prior to DSB repair. The current consensus is to use single-stranded oligonucleotide DNA templates $<200$ bases in length for small insertions such as epitope tags or to install particular gene mutations. Homology arm sizes can range from approximately 30-90 bases, 50-80 being common. Single-stranded DNA, as opposed to doublestranded, reduces the risk of integration at other genomic positions by sequence-independent endligation reactions. For longer insertions, single-stranded DNA donor sequences can be synthesized, for example the Easi-CRISPR method uses in vitro transcription followed by reverse transcription for template preparation [79]. More often though, when adding a fluorescent protein fusion to an endogenous coding sequence, methods have resorted to double-stranded plasmid DNA for the template. In this case, extensive $>800$ bp homology arms are needed and targeted integration rates are very low. Whatever the template type, a small number of base changes at PAM or seed sequence positions are included to ensure that a successfully modified gene is not subjected to further cleavage by the Cas9-gRNA complex.

In Xenopus, the principal reason for combining the oocyte host-transfer technique with CRISPR/Cas9 is the substantially higher homologous recombination activity reported in oocytes compared to fertilized embryos [80]. By utilizing HDR in oocytes, G0-generation embryos could be produced that harboured non-mosaic heterozygous insertion of epitope tag DNAs with error-free integration boundaries [76,77]. The insertions were detected in 4-9\% of the resulting tadpoles and this efficiency could be increased to $17-26 \%$ by incubating the oocytes with an inhibitor of DNA ligase (SCR-7) to suppress competing NHEJ repair. Nonetheless, before committing to an oocyte HDR project, it would be wise to test more recently developed innovations for long donor template production together with their simple injection into fertilized Xenopus embryos. For example, a long single-stranded DNA template containing phosphothioate modifications has achieved mosaic correction of an existing $X$. tropicalis tyr pigmentation mutation in 35\% of injected embryos. Similarly in $X$. laevis, the in-frame insertion of a fluorescent protein (sfGFP) fusion into the melanocyte-specific slc45a2 L-gene copy was also reported in $20 \%$ of the embryos [81]. Moreover, the Easi-CRISPR method in mouse zygote injections could install knock-in alleles with average efficiencies of 30-60\% (see https://blog.addgene.org/easi-crispr-generating-knock-in-andconditional-mouse-models) [79,82].

Previously, strategies for inserting DNA sequences during NHEJ were attractive because this is the most frequently used mechanism for repairing Cas9 DSBs in the early embryo. The donor template must be designed so that the new sequence will function following imprecise integration at the targeted location in the gene, since indels do occur at the insert boundaries in this method. In 
practice, this means selecting a DSB in an intron and incorporating a revised intron-exon complete with working splice junction that will replace the natural downstream exons in the mature mRNA transcript. In this way, replacement final exons have been installed into both zebrafish $[83,84]$ and $X$. tropicalis [85] genes that encode C-terminal fluorescent protein fusions. The plasmid donor template includes at least one homology arm featuring a hybridization site for the chosen gRNA (termed a bait). After co-injection with Cas9 and the gRNA, concurrent cleavage to linearize the donor template and produce the DSB at the target site aids insertion at the correct genetic location. Refinements to the donor template include having two gRNA sites placed either side of the insert DNA to release it from the plasmid backbone for cleaner installation and to add the natural 3'-UTR of the gene to improve expression [17]. However with NHEJ, there is no control over which direction a DNA sequence will be inserted, plus an equimolar fragment of liberated plasmid backbone will become a competing substrate for the ligase. These disadvantages reduce the occurrence of functional insertions. Furthermore, these whole exon knock-ins via NHEJ are only suited to editing applications towards the 3'-end of genes.

As a further improvement, template flanking arms can be designed to encourage microhomology mediated end-joining (MMEJ) rather than NHEJ repair, which will impart some control over the insertion boundary sequences and orientation of integration. This approach was originally developed for gene editing at TALENS-mediated DSBs [86] and has been modified to work during repair of CRISPR/Cas9 cuts [87]. Three different gRNAs are employed. The principal gRNA directs Cas9 cleavage of the genomic target as per usual. The second and third gRNAs are designed to cut at the left and right flanking arms to release the insert template and are arranged on opposite DNA strands with their PAMs external to the cleavage sites. Importantly, each end of the released insert includes a short region ( $~ 8 \mathrm{bp}$ ) that is identical to one of the two sequences adjacent to the genomic DSB, thus stimulating MMEJ repair with directional integration. The gRNAs are specific to their own target sites and will not hybridize to the selected gene after successful installation of the new DNA sequence. Functioning fusion protein insertions with homogeneous expression patterns suggesting integration at the one-cell stage have been reported in $15 \%$ of injected $X$. laevis embryos (for TALENS-MMEJ), with the anticipated sequence boundaries also confirmed [86]. However with this system, the true extent of allele mosaicism in embryos must be evaluated (in case multiple integrants occurred later in development) and there are now three gRNAs to be screened for offtarget mutagenic potential. Whichever strategy is selected to incorporate new DNA sequences into a Xenopus gene, there are now many published methods to provide guidance, in order to optimize insertion efficiency, precisely control sequence boundaries, speed up downstream analysis and ensure necessary controls are included.

\section{9. $X$. laevis and $X$. tropicalis are suited to different types of gene editing projects (at the moment).}

It is well known that the two laboratory Xenopus species have different strengths and this applies especially to gene editing experiments. The true diploid genome and shorter maturation time of $X$. tropicalis suits pipelines for the production and analysis of defined, deleterious gene mutations. The emphasis should be on accurate quantification of defective phenotypes as they arise in the developing embryo and study of genes whose human orthologs are implicated in congenital disease. $X$. tropicalis knock-outs have already been used as part of large, multi-species studies that confirm disease causality for candidate genes identified through patient genome sequencing $[13,88,89]$. The real challenge is to move beyond functional-nulls and to replicate the actual missense mutations found in patients, which are likely to encode far subtler hypomorphic changes to protein activity. The base or prime editing, DNA insertion and rapid analysis strategies outlined here can be linked to achieve this goal. It is these subtle mutations and the way they disturb embryonic regulatory networks that will prove most informative; understanding how such birth defects arise or how later- 
presenting adult diseases can have complex embryonic origins will justify the effort of disease modelling in Xenopus.

Both species work perfectly well for CRISPR experiments in cell and developmental biology research, including addition of fluorescent protein fusion tags and analysis of combinatorial knockout of genes by multiplexing injection of several gRNAs in G0-generation embryos. The cellular mosaicism in CRISPR/Cas9 mutated $X$. laevis embryos is acknowledged to be less complex, on average, than that observed in $X$. tropicalis [31]. In spite of its larger genome, the slower development allows longer effective time for guided nuclease injection, DSB formation and repairs to complete whilst at the one-cell stage. Plus, the large embryo size of $X$. laevis tolerates higher doses of injected reagents. Intriguingly, if the latest generation of base editors display the same activity in embryos as they have in cells, then phenotypic analysis of precisely selected gene mutations can also be performed immediately, post-injection. In this case, with careful gRNA design and deep sequencing of resulting alleles, $X$. laevis embryos also become an excellent choice for base editing-driven gene functional analyses.

\section{The education of gene editing - A new role for $X$. laevis embryos.}

The next generation of scientists, medical and healthcare professionals need educating in the technical and ethical aspects of gene editing. In truth, the whole of society does. Arguably the most important recent CRISPR paper to feature Xenopus embryos is devoted to student training. Martin, Wolcott and O'Connell [90] reveal how they devised undergraduate laboratory courses to provide students with direct experience of CRISPR/Cas9 gene knock-outs in frogs and butterflies. It is a comprehensive account that first defines the education goals they want students to achieve and then describes course design, content, how it is implemented, an accompanying lecture series, assignments, student reporting and teacher assessment. The aim is to provide a clear structure that guarantees learning outcomes whilst supporting students as they conduct their own hypothesis-led experiments (known as a course-based undergraduate research experience, or CURE).

In making their choice of experimental species, they note that, 'amphibians and lepidopteran species are charismatic animals that easily capture the attention of undergraduates, and also have long and rich histories of addressing fundamental questions in biology by cross-cutting fields of evolution, development, behavior, ecology and genetics.' They use $X$. laevis and the butterfly Vanessa cardui, species that reliably yield hundreds of large and freshly fertilized embryos in a scalable manner. $X$. laevis is the most robust and easiest vertebrate embryo to learn to micro-inject and knock-out phenotypes affecting development can be obtained within a week of practical classes. The genes chosen for mutation can be tailored to fit an overarching theme for that term's study, or fit with academic expertise of the faculty. For CUREs that target gene knock-outs that have not been tested before, students actively participate in the creation of scientific knowledge. The intricacies of gene editing design, somatic genotype sequence analysis, phenotype classification and off-target effects are all brought to life. These experimental insights also stimulate a deeper study of the bioethical challenges raised by gene editing. The authors argue that 'Science-Technology and Society' modules have not been given sufficient prominence in current biology degree courses. 'Empowering students with a technical understanding of genome editing comes with the obligation of bioethics discussions', and so detail how these are integrated into their laboratory course. Is teaching a future career for frogs? Overall, a compelling case for $X$. laevis embryos in gene editing education is presented that can be widely adopted among institutions with access to an aquatics facility.

\section{Concluding remarks.}

The purpose of this overview is to encourage use of CRISPR methods that allow greater control over the mutations, DNA insertions and deletions being installed into Xenopus genes. The benefits 
of increased precision are obvious in terms of eliminating the cellular mosaicism and thus enabling faster discovery of quantifiable results. While CRISPant mutated embryos can be obtained quickly, there is a limit to the experimental detail they can provide. The pace of technical developments in this field is rapid. A literature review that is accurate in Spring 2021 will quickly become dated and so early adoption of new editing strategies across the Xenopus community is key. Reviewing published works also highlighted variation in how thoroughly the targeted gene alleles (and offtarget effects) were confirmed by sequencing. This should be straightforward to improve. Commercial NGS costs have reduced hugely and further savings can be made if necessary by coding the amplicon primers for distinct samples and then multiplexing NGS reads [91,92].

As we have seen, transition missense mutations can be introduced using a choice of different base editors, while transversions, insertions and deletions are specified by prime editors. Cas9-targeted DSBs remain useful as openings for the insertion of longer DNA sequences including those that encode fluorescent protein fusions. Web applications automate experiment design so that a genomic sequence of interest submitted along with a request for a specific variant rapidly generates a list of candidate base editor gRNAs and/or prime editor pegRNAs/ngRNAs to produce the mutation or the reversion [74]. Algorithms that predict base editing outcomes and their efficiencies are also being actively pursued [93]. Software does not yet include all available editors; there is less support for CBEs and prime editors with altered PAM specificity. Hence drawing out a proposed new gene editing experiment will help illustrate the possible options. For a chosen region of gene coding sequence, visualizing both DNA strands along with its translation is a reliable way to determine what amino acid substitutions are attainable using base editors (Fig 1) and what changes will require a prime editor (Fig 3). The relative positioning of the spacer sequence with respect to the mutation is obviously different for the two strategies and all resulting guides must be screened to ensure target specificity. If a long sequence insertion is required, then a DSB cleavage site must be identified and donor template sequence produced, including choosing the type of homology arms employed (HDR or MMEJ) to aid integration.

Finally, the example gRNAs and pegRNAs depicted in Fig 1 to Fig 3 target the Xenopus adprhl1 gene. They were chosen to highlight how base and prime editing can accelerate studies of protein function. Adprhl1 plays an essential role in heart chamber muscle myofibrillogenesis and consequently CRISPant gene knock-out embryos display defective ventricle outgrowth [14]. The underlying mechanism by which Adprhl1 supports muscle growth has not yet been determined. The new editing experiments (Fig 1, Fig 3) enable rapid functional analysis of arginine residues within an ancestrally conserved substrate binding cleft that have been implicated as a source of Adprhl1's cardiac activity [14]. Of course, the prospect of a striking heart phenotype that is easily identified and linked to specific missense mutations in adprhl1 means the gRNAs/pegRNAs would be excellent candidates for testing by an undergraduate laboratory course. 


\section{References.}

1. Nieuwkoop PD, Faber J. Normal table of Xenopus laevis (Daudin): A systematical and chronological survey of the development from the fertilized egg till the end of metamorphosis. New York: Garland c1994; 1956.

2. Karimi K, Fortriede JD, Lotay VS, Burns KA, Wang DZ, Fisher ME, et al. Xenbase: a genomic, epigenomic and transcriptomic model organism database. Nucleic Acids Res. 2018;46: D861-D868. doi:10.1093/nar/gkx936

3. Bosch DL. The evolution of amphibians: the conquest of the land. In: All you need is Biology [Internet]. 6 May 2015 [cited 10 Nov 2020]. Available: https://allyouneedisbiology.wordpress.com/2015/05/07/evolutionamphibians/

4. Bosch DL. Frogs, toads and newts: the last amphibians. In: All you need is Biology [Internet]. 1 Jun 2015 [cited 10 Nov 2020]. Available: https://allyouneedisbiology.wordpress.com/2015/06/01/lissamphibia/

5. Clack JA. The Fish-Tetrapod Transition: New Fossils and Interpretations. Evo Edu Outreach. 2009;2: 213-223. doi:10.1007/s12052-009-0119-2

6. Jensen B, Wang T, Christoffels VM, Moorman AFM. Evolution and development of the building plan of the vertebrate heart. Biochim Biophys Acta. 2013;1833: 783-794. doi:10.1016/j.bbamcr.2012.10.004

7. Smith SJ, Towers N, Saldanha JW, Shang CA, Mahmood SR, Taylor WR, et al. The cardiac-restricted protein ADP-ribosylhydrolase-like 1 is essential for heart chamber outgrowth and acts on muscle actin filament assembly. Dev Biol. 2016;416: 373-388. doi:10.1016/j.ydbio.2016.05.006

8. Session AM, Uno Y, Kwon T, Chapman JA, Toyoda A, Takahashi S, et al. Genome evolution in the allotetraploid frog Xenopus laevis. Nature. 2016;538: 336-343. doi:10.1038/nature19840

9. Hellsten U, Harland RM, Gilchrist MJ, Hendrix D, Jurka J, Kapitonov V, et al. The genome of the Western clawed frog Xenopus tropicalis. Science. 2010;328: 633-636. doi:10.1126/science.1183670

10. Abu-Daya A, Khokha MK, Zimmerman LB. The hitchhiker's guide to Xenopus genetics. Genesis. 2012;50: 164175. doi:10.1002/dvg.22007

11. Jinek M, Chylinski K, Fonfara I, Hauer M, Doudna JA, Charpentier E. A programmable dual-RNA-guided DNA endonuclease in adaptive bacterial immunity. Science. 2012;337: 816-821. doi:10.1126/science.1225829

12. Sakane Y, Iida M, Hasebe T, Fujii S, Buchholz DR, Ishizuya-Oka A, et al. Functional analysis of thyroid hormone receptor beta in Xenopus tropicalis founders using CRISPR-Cas. Biol Open. 2018;7. doi:10.1242/bio.030338

13. Kariminejad A, Szenker-Ravi E, Lekszas C, Tajsharghi H, Moslemi A-R, Naert T, et al. Homozygous Null TBX4 Mutations Lead to Posterior Amelia with Pelvic and Pulmonary Hypoplasia. Am J Hum Genet. 2019;105: 12941301. doi:10.1016/j.ajhg.2019.10.013

14. Smith SJ, Towers N, Demetriou K, Mohun TJ. Defective heart chamber growth and myofibrillogenesis after knockout of adprhl1 gene function by targeted disruption of the ancestral catalytic active site. PLoS ONE. 2020;15: e0235433. doi:10.1371/journal.pone.0235433

15. Moreno-Mateos MA, Fernandez JP, Rouet R, Vejnar CE, Lane MA, Mis E, et al. CRISPR-Cpf1 mediates efficient homology-directed repair and temperature-controlled genome editing. Nat Commun. 2017;8: 2024. doi:10.1038/s41467-017-01836-2

16. Edholm E-S, Banach M, Hyoe Rhoo K, Pavelka MS, Robert J. Distinct MHC class I-like interacting invariant T cell lineage at the forefront of mycobacterial immunity uncovered in Xenopus. Proc Natl Acad Sci U S A.

2018;115: E4023-E4031. doi:10.1073/pnas.1722129115 
17. Mao C-Z, Zheng L, Zhou Y-M, Wu H-Y, Xia J-B, Liang C-Q, et al. CRISPR/Cas9-mediated efficient and precise targeted integration of donor DNA harboring double cleavage sites in Xenopus tropicalis. FASEB J. 2018; fj201800093. doi:10.1096/fj.201800093

18. Shi Z, Xin H, Tian D, Lian J, Wang J, Liu G, et al. Modeling human point mutation diseases in Xenopus tropicalis with a modified CRISPR/Cas9 system. FASEB J. 2019;33: 6962-6968. doi:10.1096/fj.201802661R

19. Viet J, Reboutier D, Hardy S, Lachke SA, Paillard L, Gautier-Courteille C. Modeling ocular lens disease in Xenopus. Dev Dyn. 2020;249: 610-621. doi:10.1002/dvdy.147

20. DeLay BD, Baldwin TA, Miller RK. Dynamin Binding Protein Is Required for Xenopus laevis Kidney Development. Front Physiol. 2019;10: 143. doi:10.3389/fphys.2019.00143

21. Wen RH, Stanar P, Tam B, Moritz OL. Autophagy in Xenopus laevis rod photoreceptors is independently regulated by phototransduction and misfolded RHOP23H. Autophagy. 2019;15: 1970-1989. doi:10.1080/15548627.2019.1596487

22. Bharathan NK, Dickinson AJG. Desmoplakin is required for epidermal integrity and morphogenesis in the Xenopus laevis embryo. Dev Biol. 2019;450: 115-131. doi:10.1016/j.ydbio.2019.03.010

23. Date P, Ackermann P, Furey C, Fink IB, Jonas S, Khokha MK, et al. Visualizing flow in an intact CSF network using optical coherence tomography: implications for human congenital hydrocephalus. Sci Rep. 2019;9: 6196. doi:10.1038/s41598-019-42549-4

24. Lalonde S, Stone OA, Lessard S, Lavertu A, Desjardins J, Beaudoin M, et al. Frameshift indels introduced by genome editing can lead to in-frame exon skipping. PLoS One. 2017;12: e0178700. doi:10.1371/journal.pone.0178700

25. Mou H, Smith JL, Peng L, Yin H, Moore J, Zhang X-O, et al. CRISPR/Cas9-mediated genome editing induces exon skipping by alternative splicing or exon deletion. Genome Biol. 2017;18: 108. doi:10.1186/s13059-017$1237-8$

26. Blitz IL, Fish MB, Cho KWY. Leapfrogging: primordial germ cell transplantation permits recovery of CRISPR/Cas9-induced mutations in essential genes. Development. 2016;143: 2868-2875. doi:10.1242/dev.138057

27. Shi J, Wang E, Milazzo JP, Wang Z, Kinney JB, Vakoc CR. Discovery of cancer drug targets by CRISPR-Cas9 screening of protein domains. Nat Biotechnol. 2015;33: 661-667. doi:10.1038/nbt.3235

28. Schaefer M, Clevert D-A, Weiss B, Steffen A. PAVOOC: designing CRISPR sgRNAs using 3D protein structures and functional domain annotations. Bioinformatics. 2019;35: 2309-2310. doi:10.1093/bioinformatics/bty935

29. Ceccaldi R, Liu JC, Amunugama R, Hajdu I, Primack B, Petalcorin MIR, et al. Homologous-recombinationdeficient tumours are dependent on PolO-mediated repair. Nature. 2015;518: 258-262. doi:10.1038/nature14184

30. Seol J-H, Shim EY, Lee SE. Microhomology-mediated end joining: Good, bad and ugly. Mutat Res. 2018;809: 81-87. doi:10.1016/j.mrfmmm.2017.07.002

31. Naert T, Tulkens D, Edwards NA, Carron M, Shaidani N-I, Wlizla M, et al. Maximizing CRISPR/Cas9 phenotype penetrance applying predictive modeling of editing outcomes in Xenopus and zebrafish embryos. Scientific Reports. 2020;10: 14662. doi:10.1038/s41598-020-71412-0

32. Komor AC, Kim YB, Packer MS, Zuris JA, Liu DR. Programmable editing of a target base in genomic DNA without double-stranded DNA cleavage. Nature. 2016;533: 420-424. doi:10.1038/nature17946

33. Harris RS, Petersen-Mahrt SK, Neuberger MS. RNA editing enzyme APOBEC1 and some of its homologs can act as DNA mutators. Mol Cell. 2002;10: 1247-1253. doi:10.1016/s1097-2765(02)00742-6 
34. Kunz C, Saito Y, Schär P. DNA Repair in mammalian cells: Mismatched repair: variations on a theme. Cell Mol Life Sci. 2009;66: 1021-1038. doi:10.1007/s00018-009-8739-9

35. Mol CD, Arvai AS, Sanderson RJ, Slupphaug G, Kavli B, Krokan HE, et al. Crystal structure of human uracilDNA glycosylase in complex with a protein inhibitor: protein mimicry of DNA. Cell. 1995;82: 701-708. doi:10.1016/0092-8674(95)90467-0

36. Nishida K, Arazoe T, Yachie N, Banno S, Kakimoto M, Tabata M, et al. Targeted nucleotide editing using hybrid prokaryotic and vertebrate adaptive immune systems. Science. 2016;353. doi:10.1126/science.aaf8729

37. Gaudelli NM, Komor AC, Rees HA, Packer MS, Badran AH, Bryson DI, et al. Programmable base editing of A •T to $\mathrm{G} \cdot \mathrm{C}$ in genomic DNA without DNA cleavage. Nature. 2017;551: 464-471. doi:10.1038/nature24644

38. Kim J, Malashkevich V, Roday S, Lisbin M, Schramm VL, Almo SC. Structural and kinetic characterization of Escherichia coli TadA, the wobble-specific tRNA deaminase. Biochemistry. 2006;45: 6407-6416. doi:10.1021/bi0522394

39. Thuronyi BW, Koblan LW, Levy JM, Yeh W-H, Zheng C, Newby GA, et al. Continuous evolution of base editors with expanded target compatibility and improved activity. Nat Biotechnol. 2019;37: 1070-1079. doi:10.1038/s41587-019-0193-0

40. Rees HA, Komor AC, Yeh W-H, Caetano-Lopes J, Warman M, Edge ASB, et al. Improving the DNA specificity and applicability of base editing through protein engineering and protein delivery. Nat Commun. 2017;8: 15790. doi:10.1038/ncomms15790

41. Richter MF, Zhao KT, Eton E, Lapinaite A, Newby GA, Thuronyi BW, et al. Phage-assisted evolution of an adenine base editor with improved Cas domain compatibility and activity. Nat Biotechnol. 2020;38: 883-891. doi:10.1038/s41587-020-0453-z

42. Zuo E, Sun Y, Wei W, Yuan T, Ying W, Sun H, et al. Cytosine base editor generates substantial off-target singlenucleotide variants in mouse embryos. Science. 2019;364: 289-292. doi:10.1126/science.aav9973

43. Jin S, Zong Y, Gao Q, Zhu Z, Wang Y, Qin P, et al. Cytosine, but not adenine, base editors induce genome-wide off-target mutations in rice. Science. 2019;364: 292-295. doi:10.1126/science.aaw7166

44. McGrath E, Shin H, Zhang L, Phue J-N, Wu WW, Shen R-F, et al. Targeting specificity of APOBEC-based cytosine base editor in human iPSCs determined by whole genome sequencing. Nat Commun. 2019;10: 5353. doi:10.1038/s41467-019-13342-8

45. Doman JL, Raguram A, Newby GA, Liu DR. Evaluation and minimization of Cas9-independent off-target DNA editing by cytosine base editors. Nat Biotechnol. 2020;38: 620-628. doi:10.1038/s41587-020-0414-6

46. Grünewald J, Zhou R, Garcia SP, Iyer S, Lareau CA, Aryee MJ, et al. Transcriptome-wide off-target RNA editing induced by CRISPR-guided DNA base editors. Nature. 2019;569: 433-437. doi:10.1038/s41586-019-1161-z

47. Zhou C, Sun Y, Yan R, Liu Y, Zuo E, Gu C, et al. Off-target RNA mutation induced by DNA base editing and its elimination by mutagenesis. Nature. 2019;571: 275-278. doi:10.1038/s41586-019-1314-0

48. Rees HA, Wilson C, Doman JL, Liu DR. Analysis and minimization of cellular RNA editing by DNA adenine base editors. Sci Adv. 2019;5: eaax5717. doi:10.1126/sciadv.aax5717

49. Anzalone AV, Koblan LW, Liu DR. Genome editing with CRISPR-Cas nucleases, base editors, transposases and prime editors. Nat Biotechnol. 2020;38: 824-844. doi:10.1038/s41587-020-0561-9

50. Zhang Y, Qin W, Lu X, Xu J, Huang H, Bai H, et al. Programmable base editing of zebrafish genome using a modified CRISPR-Cas9 system. Nat Commun. 2017;8: 118. doi:10.1038/s41467-017-00175-6 
51. Park D-S, Yoon M, Kweon J, Jang A-H, Kim Y, Choi S-C. Targeted Base Editing via RNA-Guided Cytidine Deaminases in Xenopus laevis Embryos. Mol Cells. 2017;40: 823-827. doi:10.14348/molcells.2017.0262

52. Kim K, Ryu S-M, Kim S-T, Baek G, Kim D, Lim K, et al. Highly efficient RNA-guided base editing in mouse embryos. Nat Biotechnol. 2017;35: 435-437. doi:10.1038/nbt.3816

53. Sasaguri H, Nagata K, Sekiguchi M, Fujioka R, Matsuba Y, Hashimoto S, et al. Introduction of pathogenic mutations into the mouse Psen1 gene by Base Editor and Target-AID. Nat Commun. 2018;9: 2892. doi:10.1038/s41467-018-05262-w

54. Levy JM, Yeh W-H, Pendse N, Davis JR, Hennessey E, Butcher R, et al. Cytosine and adenine base editing of the brain, liver, retina, heart and skeletal muscle of mice via adeno-associated viruses. Nat Biomed Eng. 2020;4: 97110. doi:10.1038/s41551-019-0501-5

55. Koblan LW, Erdos MR, Wilson C, Cabral WA, Levy JM, Xiong Z-M, et al. In vivo base editing rescues Hutchinson-Gilford progeria syndrome in mice. Nature. 2021. doi:10.1038/s41586-020-03086-7

56. Song C-Q, Jiang T, Richter M, Rhym LH, Koblan LW, Zafra MP, et al. Adenine base editing in an adult mouse model of tyrosinaemia. Nat Biomed Eng. 2020;4: 125-130. doi:10.1038/s41551-019-0357-8

57. Ryu S-M, Koo T, Kim K, Lim K, Baek G, Kim S-T, et al. Adenine base editing in mouse embryos and an adult mouse model of Duchenne muscular dystrophy. Nat Biotechnol. 2018;36: 536-539. doi:10.1038/nbt.4148

58. Villiger L, Grisch-Chan HM, Lindsay H, Ringnalda F, Pogliano CB, Allegri G, et al. Treatment of a metabolic liver disease by in vivo genome base editing in adult mice. Nat Med. 2018;24: 1519-1525. doi:10.1038/s41591018-0209-1

59. Jiang T, Henderson JM, Coote K, Cheng Y, Valley HC, Zhang X-O, et al. Chemical modifications of adenine base editor mRNA and guide RNA expand its application scope. Nat Commun. 2020;11: 1979. doi:10.1038/s41467020-15892-8

60. Liu Z, Chen M, Chen S, Deng J, Song Y, Lai L, et al. Highly efficient RNA-guided base editing in rabbit. Nat Commun. 2018;9: 2717. doi:10.1038/s41467-018-05232-2

61. Anzalone AV, Randolph PB, Davis JR, Sousa AA, Koblan LW, Levy JM, et al. Search-and-replace genome editing without double-strand breaks or donor DNA. Nature. 2019;576: 149-157. doi:10.1038/s41586-019-17114

62. Liu Y, Kao H-I, Bambara RA. Flap endonuclease 1: a central component of DNA metabolism. Annu Rev Biochem. 2004;73: 589-615. doi:10.1146/annurev.biochem.73.012803.092453

63. Keijzers G, Bohr VA, Rasmussen LJ. Human exonuclease 1 (EXO1) activity characterization and its function on flap structures. Biosci Rep. 2015;35. doi:10.1042/BSR20150058

64. Liu P, Liang S-Q, Zheng C, Mintzer E, Zhao YG, Ponnienselvan K, et al. Improved prime editors enable pathogenic allele correction and cancer modelling in adult mice. Nat Commun. 2021;12: 2121. doi:10.1038/s41467-021-22295-w

65. Kweon J, Yoon J-K, Jang A-H, Shin HR, See J-E, Jang G, et al. Engineered prime editors with PAM flexibility. Mol Ther. 2021. doi:10.1016/j.ymthe.2021.02.022

66. Kim DY, Moon SB, Ko J-H, Kim Y-S, Kim D. Unbiased investigation of specificities of prime editing systems in human cells. Nucleic Acids Res. 2020;48: 10576-10589. doi:10.1093/nar/gkaa764

67. Jin S, Lin Q, Luo Y, Zhu Z, Liu G, Li Y, et al. Genome-wide specificity of prime editors in plants. Nat Biotechnol. 2021. doi:10.1038/s41587-021-00891-x 
68. Sürün D, Schneider A, Mircetic J, Neumann K, Lansing F, Paszkowski-Rogacz M, et al. Efficient Generation and Correction of Mutations in Human iPS Cells Utilizing mRNAs of CRISPR Base Editors and Prime Editors. Genes (Basel). 2020;11. doi:10.3390/genes11050511

69. Schene IF, Joore IP, Oka R, Mokry M, van Vugt AHM, van Boxtel R, et al. Prime editing for functional repair in patient-derived disease models. Nat Commun. 2020;11: 5352. doi:10.1038/s41467-020-19136-7

70. Liu Y, Li X, He S, Huang S, Li C, Chen Y, et al. Efficient generation of mouse models with the prime editing system. Cell Discov. 2020;6: 27. doi:10.1038/s41421-020-0165-z

71. Gao P, Lyu Q, Ghanam AR, Lazzarotto CR, Newby GA, Zhang W, et al. Prime editing in mice reveals the essentiality of a single base in driving tissue-specific gene expression. Genome Biol. 2021;22: 83. doi:10.1186/s13059-021-02304-3

72. Bosch JA, Birchak G, Perrimon N. Precise genome engineering in Drosophila using prime editing. Proc Natl Acad Sci U S A. 2021;118. doi:10.1073/pnas.2021996118

73. Hsu JY, Grünewald J, Szalay R, Shih J, Anzalone AV, Lam KC, et al. PrimeDesign software for rapid and simplified design of prime editing guide RNAs. Nat Commun. 2021;12: 1034. doi:10.1038/s41467-021-21337-7

74. Siegner SM, Karasu ME, Schröder MS, Kontarakis Z, Corn JE. PnB Designer: a web application to design prime and base editor guide RNAs for animals and plants. BMC Bioinformatics. 2021;22: 101. doi:10.1186/s12859021-04034-6

75. Blitz IL. Primordial Germ Cell Transplantation for CRISPR/Cas9-based Leapfrogging in Xenopus. J Vis Exp. 2018. doi:10.3791/56035

76. Aslan Y, Tadjuidje E, Zorn AM, Cha S-W. High-efficiency non-mosaic CRISPR-mediated knock-in and indel mutation in F0 Xenopus. Development. 2017;144: 2852-2858. doi:10.1242/dev.152967

77. Tadjuidje E, Cha S-W. How to Generate Non-Mosaic CRISPR/Cas9 Mediated Knock-In and Mutations in F0 Xenopus Through the Host-Transfer Technique. Methods Mol Biol. 2018;1865: 105-117. doi:10.1007/978-14939-8784-9_8

78. Cortez C. CRISPR 101: Homology Directed Repair. [cited 10 Dec 2020]. Available: https://blog.addgene.org/crispr-101-homology-directed-repair

79. Quadros RM, Miura H, Harms DW, Akatsuka H, Sato T, Aida T, et al. Easi-CRISPR: a robust method for onestep generation of mice carrying conditional and insertion alleles using long ssDNA donors and CRISPR ribonucleoproteins. Genome Biol. 2017;18: 92. doi:10.1186/s13059-017-1220-4

80. Hagmann M, Adlkofer K, Pfeiffer P, Bruggmann R, Georgiev O, Rungger D, et al. Dramatic changes in the ratio of homologous recombination to nonhomologous DNA-end joining in oocytes and early embryos of Xenopus laevis. Biol Chem Hoppe Seyler. 1996;377: 239-250. doi:10.1515/bchm3.1996.377.4.239

81. Nakayama T, Grainger RM, Cha S-W. Simple embryo injection of long single-stranded donor templates with the CRISPR/Cas9 system leads to homology-directed repair in Xenopus tropicalis and Xenopus laevis. Genesis. 2020;58: e23366. doi:10.1002/dvg.23366

82. Gearing M. Easi-CRISPR: Generating Knock-In and Conditional Mouse Models. [cited 10 Dec 2020]. Available: https://blog.addgene.org/easi-crispr-generating-knock-in-and-conditional-mouse-models

83. Li J, Zhang B, Ren Y, Gu S, Xiang Y, Du J. Intron targeting-mediated and endogenous gene integrity-maintaining knockin in zebrafish using the CRISPR/Cas9 system. Cell Res. 2015;25: 634-637. doi:10.1038/cr.2015.43

84. Li J, Zhang B, Bu J, Du J. Intron-based genomic editing: a highly efficient method for generating knockin zebrafish. Oncotarget. 2015;6: 17891-17894. doi:10.18632/oncotarget.4547 
85. Shi Z, Wang F, Cui Y, Liu Z, Guo X, Zhang Y, et al. Heritable CRISPR/Cas9-mediated targeted integration in Xenopus tropicalis. FASEB J. 2015;29: 4914-4923. doi:10.1096/fj.15-273425

86. Nakade S, Tsubota T, Sakane Y, Kume S, Sakamoto N, Obara M, et al. Microhomology-mediated end-joiningdependent integration of donor DNA in cells and animals using TALENs and CRISPR/Cas9. Nat Commun. 2014;5: 5560. doi:10.1038/ncomms6560

87. Suzuki K-IT, Sakane Y, Suzuki M, Yamamoto T. A Simple Knock-In System for Xenopus via Microhomology Mediated End Joining Repair. Methods Mol Biol. 2018;1865: 91-103. doi:10.1007/978-1-4939-8784-9_7

88. Landim-Vieira M, Johnston JR, Ji W, Mis EK, Tijerino J, Spencer-Manzon M, et al. Familial Dilated Cardiomyopathy Associated With a Novel Combination of Compound Heterozygous TNNC1 Variants. Front Physiol. 2019;10: 1612. doi:10.3389/fphys.2019.01612

89. Alharatani R, Ververi A, Beleza-Meireles A, Ji W, Mis E, Patterson QT, et al. Novel truncating mutations in CTNND1 cause a dominant craniofacial and cardiac syndrome. Hum Mol Genet. 2020;29: 1900-1921. doi:10.1093/hmg/ddaa050

90. Martin A, Wolcott NS, O’Connell LA. Bringing immersive science to undergraduate laboratory courses using CRISPR gene knockouts in frogs and butterflies. J Exp Biol. 2020;223. doi:10.1242/jeb.208793

91. Iida M, Suzuki M, Sakane Y, Nishide H, Uchiyama I, Yamamoto T, et al. A simple and practical workflow for genotyping of CRISPR-Cas9-based knockout phenotypes using multiplexed amplicon sequencing. Genes Cells. 2020;25: 498-509. doi:10.1111/gtc.12775

92. Steyaert W, Boel A, Coucke P, Willaert A. BATCH-GE: Analysis of NGS Data for Genome Editing Assessment. Methods Mol Biol. 2018;1865: 83-90. doi:10.1007/978-1-4939-8784-9_6

93. Arbab M, Shen MW, Mok B, Wilson C, Matuszek Ż, Cassa CA, et al. Determinants of Base Editing Outcomes from Target Library Analysis and Machine Learning. Cell. 2020;182: 463-480.e30. doi:10.1016/j.cell.2020.05.037

94. Zahn N, Levin M, Adams DS. The Zahn drawings: new illustrations of Xenopus embryo and tadpole stages for studies of craniofacial development. Development. 2017;144: 2708-2713. doi:10.1242/dev.151308

95. Wiechmann AF, Martin TA, Horb ME. CRISPR/Cas9 mediated mutation of the mtnr1a melatonin receptor gene causes rod photoreceptor degeneration in developing Xenopus tropicalis. Sci Rep. 2020;10: 13757. doi:10.1038/s41598-020-70735-2

96. Wang C, Qi X, Zhou X, Sun J, Cai D, Lu G, et al. RNA-Seq analysis on ets1 mutant embryos of Xenopus tropicalis identifies microseminoprotein beta gene 3 as an essential regulator of neural crest migration. FASEB J. 2020. doi:10.1096/fj.202000603R

97. Shibata Y, Tanizaki Y, Shi Y-B. Thyroid hormone receptor beta is critical for intestinal remodeling during Xenopus tropicalis metamorphosis. Cell Biosci. 2020;10: 46. doi:10.1186/s13578-020-00411-5

98. Kodama M, Yoshida M, Endo M, Kobayashi T, Oike A, Yasumasu S, et al. Nanos3 of the frog Rana rugosa: Molecular cloning and characterization. Dev Growth Differ. 2018;60: 112-120. doi:10.1111/dgd.12421

99. Komor AC, Zhao KT, Packer MS, Gaudelli NM, Waterbury AL, Koblan LW, et al. Improved base excision repair inhibition and bacteriophage Mu Gam protein yields C:G-to-T:A base editors with higher efficiency and product purity. Sci Adv. 2017;3: eaao4774. doi:10.1126/sciadv.aao4774

100. Koblan LW, Doman JL, Wilson C, Levy JM, Tay T, Newby GA, et al. Improving cytidine and adenine base editors by expression optimization and ancestral reconstruction. Nat Biotechnol. 2018;36: 843-846. doi:10.1038/nbt.4172 
101. Kim YB, Komor AC, Levy JM, Packer MS, Zhao KT, Liu DR. Increasing the genome-targeting scope and precision of base editing with engineered Cas9-cytidine deaminase fusions. Nat Biotechnol. 2017;35: 371-376. doi:10.1038/nbt.3803

102. Hu JH, Miller SM, Geurts MH, Tang W, Chen L, Sun N, et al. Evolved Cas9 variants with broad PAM compatibility and high DNA specificity. Nature. 2018;556: 57-63. doi:10.1038/nature26155

103. Nishimasu H, Shi X, Ishiguro S, Gao L, Hirano S, Okazaki S, et al. Engineered CRISPR-Cas9 nuclease with expanded targeting space. Science. 2018;361: 1259-1262. doi:10.1126/science.aas9129

104. Huang TP, Zhao KT, Miller SM, Gaudelli NM, Oakes BL, Fellmann C, et al. Circularly permuted and PAMmodified Cas9 variants broaden the targeting scope of base editors. Nat Biotechnol. 2019;37: 626-631. doi:10.1038/s41587-019-0134-y

105. Miller SM, Wang T, Randolph PB, Arbab M, Shen MW, Huang TP, et al. Continuous evolution of SpCas9 variants compatible with non-G PAMs. Nat Biotechnol. 2020;38: 471-481. doi:10.1038/s41587-020-0412-8

106. Walton RT, Christie KA, Whittaker MN, Kleinstiver BP. Unconstrained genome targeting with near-PAMless engineered CRISPR-Cas9 variants. Science. 2020;368: 290-296. doi:10.1126/science.aba8853

107. Zafra MP, Schatoff EM, Katti A, Foronda M, Breinig M, Schweitzer AY, et al. Optimized base editors enable efficient editing in cells, organoids and mice. Nat Biotechnol. 2018;36: 888-893. doi:10.1038/nbt.4194

108. Kleinstiver BP, Sousa AA, Walton RT, Tak YE, Hsu JY, Clement K, et al. Engineered CRISPR-Cas12a variants with increased activities and improved targeting ranges for gene, epigenetic and base editing. Nat Biotechnol. 2019;37: 276-282. doi:10.1038/s41587-018-0011-0 